\title{
On the representation of cardinalities of interval-valued fuzzy sets - the valuation property
}

\author{
Glad Deschrijver \\ Department of Mathematics and Computer Science \\ Ghent University \\ Fuzziness and Uncertainty Modelling Research Unit \\ Krijgslaan 281 (S9), B-9000 Gent \\ Belgium \\ E-mail: Glad.Deschrijver@UGent.be \\ Pavol Král' \\ Department of Quantitative Methods and Information Systems \\ Faculty of Economics \\ Matej Bel University \\ Tajovského 10, 97590 Banská Bystrica \\ Slovakia \\ E-mail: pavol.kral@umb.sk
}

\begin{abstract}
In our earlier work we have developed the axiomatic theory of the scalar cardinality of interval-valued fuzzy sets following Wygralak's axiomatic theory of scalar cardinalities of fuzzy sets. The cardinality has been defined as a mapping from the set of interval-valued fuzzy sets with finite support to the set of closed subintervals of $[0,+\infty)$. We have shown that each scalar cardinality of interval-valued fuzzy set can be characterized using the appropriate mapping called cardinality pattern. Moreover, we have found some basic conditions under which the valuation property, the subadditivity property, the complementarity rule and the cartesian product rule are satisfied using different cardinality patterns tnorms, t-conorms and negations on the lattice $\mathcal{L}^{I}$ (the underlying lattice of interval-valued fuzzy set theory). The presented paper is the first from the collection of papers consisting of the further investigation of the proposed theory providing the description of cardinality patterns, t-norms, t-conorms and negations satisfying the above mentioned properties. We restrict ourselves to the valuation property here.
\end{abstract}

Keywords: cardinality, interval-valued fuzzy set, cardinality pattern, 
valuation property

\section{Introduction}

The cardinality is a very important characteristic of crisp sets. The concept of cardinality for finite crisp sets is easily understandable and used by researchers in different research areas. Nevertheless, in some cases we are interested in simultaneous modeling of vagueness (gradations in the membership degree) and uncertainty (lack of information), e.g. we need to assess the linguistically quantified propositions (fuzzy querying in databases, expert systems, evaluation of natural language statements, aggregation, decision making in fuzzy environment, metrical analysis of grey images, etc.). For such situations we can use interval-valued fuzzy sets and we need a related concept of cardinality. There are two basic ways how to generalize the notion of cardinality for (intervalvalued) fuzzy sets - a scalar cardinality (a single number or interval), see e.g. in $[6,15,14,16,12,18,19,20,21,3,5]$, and a fuzzy cardinality (a fuzzy or interval-valued fuzzy set), see e.g. in $[21,4,17]$. The historical remarks related to the development of the concept of cardinalities of (interval-valued) fuzzy sets can be found in [11].

Following Wygralaks approach [21] we have introduced in [11] the axiomatic definition of scalar cardinalities for interval-valued fuzzy sets. We have considered the notion of cardinality as an approximation of the number of elements of a finite (interval-valued) fuzzy set. The motivation for such approach is quite straightforward. It is trivial that a cardinality of a finite crisp set can be expressed as a sum of values of the corresponding characteristic function. Further, characteristic functions can be seen as a special case of fuzzy set membership functions. Therefore it seems natural to take elements belonging to the support of a fuzzy set and sum their (transformed) membership grades to get its cardinality, a real number in general. A real number can be estimated using an appropriate interval of real numbers. So, because an interval-valued fuzzy set can be assumed as an approximation of a fuzzy set, it is quite natural to introduce cardinalities of interval-valued fuzzy sets as intervals of real numbers. The lower limit of such interval can be seen as an pessimistic and the upper limit as an optimistic evaluation of the number of elements of an (interval-valued) fuzzy set, respectively. Assuming the ordering of intervals based on comparison of interval limits and the fact that the cardinalities of crisp and fuzzy sets have to be special cases of cardinalities of interval-valued fuzzy sets, we get that cardinalities of interval-valued fuzzy sets form a special lattice.

We have also shown in [11] that each scalar cardinality of interval-valued fuzzy set can be characterized using the appropriate mapping called cardinality pattern. Moreover, we have found some basic conditions under which the valuation property, the subadditivity property, the complementarity rule and the cartesian product rule are satisfied using different cardinality patterns, t-norms, t-conorms and negations on the lattice $\mathcal{L}^{I}$ (the underlying lattice of intervalvalued fuzzy set theory). These properties have been simply adopted from the 
crisp case because, in our opinion, they can be crucial for possible practical applications. From the same point of view it is necessary to have at least a partial description of cardinalities satisfying the above mentioned properties for.

In the presented paper we restrict ourselves to the valuation property and provide the description of cardinality patterns, t-norms, t-conorms and negations satisfying it. Such description can simplify selection of appropriate cardinalities for a particular application. Since Atanassov's intuitionistic fuzzy set theory $[1,2]$ is equivalent to interval-valued fuzzy set theory [10], all results presented in this paper can be straightforwardly transformed to intuitionistic fuzzy set theory.

The paper is organized as follows. In Section 2 we present some basic preliminaries connected to interval-valued fuzzy sets, scalar cardinalities, cardinality patterns, t-norms and related operations on $\mathcal{L}^{I}$. There we also briefly mention some results from [11]. In Section 3 we study the valuation property with respect to the generalized pseudo-t-representable t-norms and t-conorms. In Section 4 and Section 5 we discuss the connection between the valuation property and the pseudo-t-representable t-norms and t-conorms of the first and the second kind, respectively. Finally, in Section 6 we focus on the valuation property and t-representable t-norms and t-conorms.

\section{Preliminary definitions}

This section consists of basic notions, definitions and properties of scalar cardinalities used in [11] in order to make this work self-contained. Throughout the paper, let $U$ denote a universal set.

\section{$2.1 \quad$ The lattice $\mathcal{L}^{I}$}

Definition 2.1 [8] We define $\mathcal{L}^{I}=\left(L^{I}, \leq_{L^{I}}\right)$ by

$$
\begin{aligned}
& L^{I}=\left\{\left[x_{1}, x_{2}\right] \mid\left(x_{1}, x_{2}\right) \in[0,1]^{2} \text { and } x_{1} \leq x_{2}\right\}, \\
& {\left[x_{1}, x_{2}\right] \leq_{L^{I}}\left[y_{1}, y_{2}\right] \Longleftrightarrow\left(x_{1} \leq y_{1} \text { and } x_{2} \leq y_{2}\right), \text { for all }\left[x_{1}, x_{2}\right],\left[y_{1}, y_{2}\right] \in L^{I} .}
\end{aligned}
$$

In the sequel, if $x \in L^{I}$, then we denote its bounds by $x_{1}$ and $x_{2}$, i.e. $x=\left[x_{1}, x_{2}\right]$. The length of the interval $x=\left[x_{1}, x_{2}\right] \in L^{I}$ is called the degree of uncertainty of $x$ and is denoted by $\pi(x)=x_{2}-x_{1}$. We define for further usage the sets

$$
\begin{aligned}
\bar{L}^{I} & =\left\{\left[x_{1}, x_{2}\right] \mid\left(x_{1}, x_{2}\right) \in \mathbb{R}^{2} \text { and } x_{1} \leq x_{2}\right\}, \\
\bar{L}_{+}^{I} & =\left\{\left[x_{1}, x_{2}\right] \mid\left(x_{1}, x_{2}\right) \in\left[0,+\infty\left[^{2} \text { and } x_{1} \leq x_{2}\right\},\right.\right. \\
D & =\{[x, x] \mid x \in[0,1]\} .
\end{aligned}
$$

For all $a \in \mathbb{R}$, we define $a_{\mathcal{L}^{I}} \in \bar{L}^{I}$ as $a_{\mathcal{L}^{I}}=[a, a]$, e.g. the smallest and the largest element of $\mathcal{L}^{I}$ are given by $0_{\mathcal{L}^{I}}=[0,0]$ and $1_{\mathcal{L}^{I}}=[1,1]$. We also extend $\leq_{L^{I}}$ to $\bar{L}^{I}$ as follows: for $x, y$ in $\bar{L}^{I}, x \leq_{\bar{L}^{I}} y \Longleftrightarrow x_{1} \leq y_{1}$ and $x_{2} \leq y_{2}$. 
Definition 2.2 [13] Let $\mathcal{L}=\left(L, \leq_{L}\right)$ be a complete lattice. An $\mathcal{L}$-fuzzy set on $U$ is a mapping $A: U \rightarrow L$.

Clearly interval-valued fuzzy sets are $\mathcal{L}^{I}$-fuzzy sets since $\mathcal{L}^{I}$ is a complete lattice. From now on, we will denote the class of interval-valued fuzzy sets on $U$ by $\mathcal{F}_{\mathcal{L}^{I}}(U)$. Let $A \in \mathcal{F}_{\mathcal{L}^{I}}(U)$, then we define

$$
\begin{aligned}
\operatorname{supp}(A) & =\left\{u \mid u \in U \text { and } A(u) \neq 0_{\mathcal{L}^{I}}\right\}, \\
\operatorname{core}(A) & =\left\{u \mid u \in U \text { and } A(u)=1_{\mathcal{L}^{I}}\right\} .
\end{aligned}
$$

If $A$ is a crisp or fuzzy set in $U$, then we will identify $A$ with the associated interval-valued fuzzy set.

An interval-valued fuzzy set $A$ on $U$ with finite support will be called a finite interval-valued fuzzy set. The class of all finite interval-valued fuzzy sets on $U$ will be denoted by $\mathcal{F}_{\mathcal{L}^{I}}^{F}(U)$.

We will use only interval-valued fuzzy sets with finite support in the rest of our paper.

Finally, we define the constant interval-valued fuzzy set $\bar{a}_{\mathcal{L}^{I}}$ by $\bar{a}_{\mathcal{L}^{I}}(u)=a_{\mathcal{L}^{I}}$, for all $u \in U$.

\subsection{Triangular norms and related operations on $\mathcal{L}^{I}$}

\section{Definition 2.3 [9]}

- A t-norm on $\mathcal{L}^{I}$ is a commutative, associative mapping $\mathcal{T}:\left(L^{I}\right)^{2} \rightarrow L^{I}$ which is increasing in both arguments and which satisfies $\mathcal{T}\left(1_{\mathcal{L}^{I}}, x\right)=x$, for all $x \in L^{I}$.

- A t-conorm on $\mathcal{L}^{I}$ is a commutative, associative mapping $\mathcal{S}:\left(L^{I}\right)^{2} \rightarrow L^{I}$ which is increasing in both arguments and which satisfies $\mathcal{S}\left(0_{\mathcal{L}^{I}}, x\right)=x$, for all $x \in L^{I}$.

- A negation on $\mathcal{L}^{I}$ is a decreasing mapping $\mathcal{N}: L^{I} \rightarrow L^{I}$ which satisfies $\mathcal{N}\left(0_{\mathcal{L}^{I}}\right)=1_{\mathcal{L}^{I}}$ and $\mathcal{N}\left(1_{\mathcal{L}^{I}}\right)=0_{\mathcal{L}^{I}}$. If $\mathcal{N}(\mathcal{N}(x))=x$, for all $x \in L^{I}$, then $\mathcal{N}$ is called involutive.

Theorem 2.4 [9] A negation $\mathcal{N}$ on $\mathcal{L}^{I}$ is involutive iff there exists an involutive negation $N$ on $[0,1]$ such that, for all $x \in L^{I}$,

$$
\mathcal{N}(x)=\left[N\left(x_{2}\right), N\left(x_{1}\right)\right] .
$$

From now on, if a negation $\mathcal{N}$ on $\mathcal{L}^{I}$ can be represented by (1) using a negation $N$ on $[0,1]$, then we call $N$ the representant of $\mathcal{N}$.

The mapping $\mathcal{N}_{s}: L^{I} \rightarrow L^{I}$ defined by, for all $x \in L^{I}$,

$$
\mathcal{N}_{s}(x)=\left[1-x_{2}, 1-x_{1}\right]
$$

is a negation on $\mathcal{L}^{I}$ which is called the standard negation on $\mathcal{L}^{I}$. Note that $\mathcal{N}_{s}(x)=\left[N_{s}\left(x_{2}\right), N_{s}\left(x_{1}\right)\right]$, for all $x \in L^{I}$, where $N_{s}$ is the standard negation on $[0,1]$ given by $N_{s}(x)=1-x$, for all $x \in[0,1]$. 
Lemma 2.5 [11] Let $\mathcal{T}$ be a t-norm and $\mathcal{N}$ an involutive negation on $\mathcal{L}^{I}$. Then the mapping $\mathcal{T}_{\mathcal{N}}:\left(L^{I}\right)^{2} \rightarrow L^{I}$ defined by, for all $x, y \in L^{I}, \mathcal{T}_{\mathcal{N}}(x, y)=$ $\mathcal{N}(\mathcal{T}(\mathcal{N}(x), \mathcal{N}(y)))$, is a t-conorm on $\mathcal{L}^{I}$.

Let $\mathcal{S}$ be a t-conorm and $\mathcal{N}$ an involutive negation on $\mathcal{L}^{I}$. Then the mapping $\mathcal{S}_{\mathcal{N}}:\left(L^{I}\right)^{2} \rightarrow L^{I}$ defined by, for all $x, y \in L^{I}, \mathcal{S}_{\mathcal{N}}(x, y)=\mathcal{N}(\mathcal{S}(\mathcal{N}(x), \mathcal{N}(y)))$, is a $t$-norm on $\mathcal{L}^{I}$.

If $\mathcal{N}=\mathcal{N}_{s}$, and $\mathcal{T}$ and $\mathcal{S}$ are a t-norm and t-conorm on $\mathcal{L}^{I}$, then we will abbreviate $\mathcal{T}_{\mathcal{N}_{s}}$ to $\mathcal{T}^{*}$ and $\mathcal{S}_{\mathcal{N}_{s}}$ to $\mathcal{S}^{*}$. Similarly, if $N=N_{s}$, and $T$ and $S$ are a t-norm and t-conorm on $[0,1]$, then we write also $T^{*}$ and $S^{*}$ instead of $T_{N_{s}}$ and $S_{N_{s}}$.

Special classes of t-norms and t-conorms are:

- the t-representable t-norms $\mathcal{T}_{T_{1}, T_{2}}$ and t-conorms $\mathcal{S}_{S_{1}, S_{2}}$ (the class $\mathcal{T}_{T_{1}, T_{2}}$ ) given by, for all $x, y \in L^{I}$,

$$
\begin{aligned}
& \mathcal{T}_{T_{1}, T_{2}}(x, y)=\left[T_{1}\left(x_{1}, y_{1}\right), T_{2}\left(x_{2}, y_{2}\right)\right], \\
& \mathcal{S}_{S_{1}, S_{2}}(x, y)=\left[S_{1}\left(x_{1}, y_{1}\right), S_{2}\left(x_{2}, y_{2}\right)\right],
\end{aligned}
$$

- the pseudo-t-representable t-norms $\mathcal{T}_{T}$ and t-conorms $\mathcal{S}_{S}$ of the first kind (the class $\mathcal{T}_{T}$ ) given by, for all $x, y \in L^{I}$,

$$
\begin{aligned}
\mathcal{T}_{T}(x, y) & =\left[T\left(x_{1}, y_{1}\right), \max \left(T\left(x_{1}, y_{2}\right), T\left(x_{2}, y_{1}\right)\right)\right], \\
\mathcal{S}_{S}(x, y) & =\left[\min \left(S\left(x_{1}, y_{2}\right), S\left(x_{2}, y_{1}\right)\right), S\left(x_{2}, y_{2}\right)\right],
\end{aligned}
$$

- the pseudo-t-representable t-norms $\mathcal{T}_{T}^{\prime}$ and t-conorms $\mathcal{S}_{S}^{\prime}$ of the second kind (the class $\mathcal{T}_{T}^{\prime}$ ) given by, for all $x, y \in L^{I}$,

$$
\begin{aligned}
\mathcal{T}_{T}^{\prime}(x, y) & =\left[\min \left(T\left(x_{1}, y_{2}\right), T\left(x_{2}, y_{1}\right)\right), T\left(x_{2}, y_{2}\right)\right], \\
\mathcal{S}_{S}^{\prime}(x, y) & =\left[S\left(x_{1}, y_{1}\right), \max \left(S\left(x_{1}, y_{2}\right), S\left(x_{2}, y_{1}\right)\right)\right],
\end{aligned}
$$

- the generalized pseudo-t-representable t-norms $\mathcal{T}_{T, t}$ and t-conorms $\mathcal{S}_{S, t}$ (the class $\mathcal{T}_{T, t}$ ) given by, for all $x, y \in L^{I}$,

$$
\begin{aligned}
& \mathcal{T}_{T, t}(x, y)=\left[T\left(x_{1}, y_{1}\right), \max \left(T\left(t, T\left(x_{2}, y_{2}\right)\right), T\left(x_{1}, y_{2}\right), T\left(x_{2}, y_{1}\right)\right)\right], \\
& \mathcal{S}_{S, t}(x, y)=\left[\min \left(S\left(1-t, S\left(x_{1}, y_{1}\right)\right), S\left(x_{1}, y_{2}\right), S\left(x_{2}, y_{1}\right)\right), S\left(x_{2}, y_{2}\right)\right],
\end{aligned}
$$

where $T_{1}, T_{2}$ and $T$ are t-norms and $S_{1}, S_{2}$ and $S$ are t-conorms on [0,1] which satisfy $T_{1} \leq T_{2}$ and $S_{1} \leq S_{2}$, and where $t$ is an arbitrary element of $[0,1]$. We call $T_{1}, T_{2}, T, S_{1}, S_{2}$ and $S$ the representants of the corresponding t-norm or t-conorm on $\mathcal{L}^{I}$. Note that if $t=0$, then the generalized pseudo-t-representable t-norm $\mathcal{T}_{T, 0}=\mathcal{T}_{T}$ is a pseudo-t-representable t-norm of the first kind, and if $t=1$, then $\mathcal{T}_{T, 1}=\mathcal{T}_{T, T}$ is a t-representable t-norm. Some examples of t-norms and t-conorms on $\mathcal{L}^{I}$ can be found in [11].

For further usage we give the following definitions. Let $\mathcal{T}$ be a t-norm on an arbitrary lattice $\mathcal{L}$ and $a \in L$, then we define $a_{\mathcal{T}}^{(1)}=a$ and $a_{\mathcal{T}}^{(n)}=\mathcal{T}\left(a, a_{\mathcal{T}}^{(n-1)}\right)$, 
for all $n \in \mathbb{N} \backslash\{0,1\}$. Let $T$ be a t-norm on $[0,1]$, then we say that $T$ is Archimedean if, for all $a, b \in] 0,1\left[\right.$, there exists $n \in \mathbb{N} \backslash\{0\}$ such that $a_{T}^{(n)}<b$. We say that $T$ has zero-divisors if there exist $a, b \in] 0,1[$ such that $T(a, b)=0$. If for an element $a \in] 0,1\left[\right.$ it holds that $a_{T}^{(n)}=0$ for some $n \in \mathbb{N} \backslash\{0\}$, then $a$ is called a nilpotent element of $T$. We say that $T$ is nilpotent if $T$ is continuous and if each $a \in] 0,1[$ is a nilpotent element of $T$.

Using t-norms, t-conorms and negations on $\mathcal{L}^{I}$, the intersection, union and complement of two interval-valued fuzzy sets $A$ and $B$ is defined as follows.

Definition 2.6 [7] The generalized intersection $\cap_{\mathcal{T}}$, union $\cup_{\mathcal{S}}$ and complement $\mathrm{co}_{\mathcal{N}}$ of interval-valued fuzzy sets is defined as follows: for all $A, B \in \mathcal{F}_{\mathcal{L}^{I}}(U)$ and for all $u \in U$,

$$
\begin{aligned}
A \cap_{\mathcal{T}} B(u) & =\mathcal{T}(A(u), B(u)), \\
A \cup_{\mathcal{S}} B(u) & =\mathcal{S}(A(u), B(u)), \\
\operatorname{co}_{\mathcal{N}} A(u) & =\mathcal{N}(A(u)) .
\end{aligned}
$$

\subsection{Scalar cardinalities of interval-valued fuzzy sets}

In [11] we have introduced the axiomatic theory of scalar cardinality of intervalvalued fuzzy sets following Wygralak's approach [21] using the following definitions of the addition on $\bar{L}^{I}$ and multiplication on $\bar{L}_{+}^{I}$ : for all $a, b \in \bar{L}^{I}$ and $c, d \in \bar{L}_{+}^{I}$,

$$
\begin{aligned}
a+b & =\left[a_{1}+b_{1}, a_{2}+b_{2}\right], \\
c \cdot d & =\left[c_{1} d_{1}, c_{2} d_{2}\right] .
\end{aligned}
$$

Definition 2.7 [11] A mapping $\operatorname{card}_{I}: \mathcal{F}_{\mathcal{L}^{I}}^{F}(U) \rightarrow \bar{L}_{+}^{I}$ is called a scalar cardinality of interval-valued fuzzy sets if the following conditions hold:

(1) coincidence: for all $u \in U$,

$$
\operatorname{card}_{I}\left(1_{\mathcal{L}^{I}} / u\right)=1_{\mathcal{L}^{I}}
$$

(2) monotonicity: for all $a, b \in L^{I}$ and $u, v \in U$,

$$
a \leq_{L^{I}} b \Longrightarrow \operatorname{card}_{I}(a / u) \leq_{L^{I}} \operatorname{card}_{I}(b / v) ;
$$

(3) additivity: for all $A, B \in \mathcal{F}_{\mathcal{L}^{I}}^{F}(U)$,

$$
\operatorname{supp}(A) \cap \operatorname{supp}(B)=\emptyset \Longrightarrow \operatorname{card}_{I}\left(A \cup_{\mathcal{S}} B\right)=\operatorname{card}_{I}(A)+\operatorname{card}_{I}(B),
$$

where $a / u$ denotes, for an arbitrary $u \in U$ and $a \in \mathcal{L}^{I}$, the interval-valued fuzzy singleton A given by

$$
A(v)= \begin{cases}a, & \text { if } v=u, \\ 0_{\mathcal{L}}, & \text { if } v \in U \backslash\{u\} .\end{cases}
$$


The following theorem brings a useful characterization of a scalar cardinality $\operatorname{card}_{I}$ :

Theorem 2.8 [11] A mapping $\operatorname{card}_{I}: \mathcal{F}_{\mathcal{L}^{I}}^{F}(U) \rightarrow \bar{L}_{+}^{I}$ is a scalar cardinality iff there exists a mapping $f_{I}: L^{I} \rightarrow L^{I}$ (called scalar cardinality pattern) fulfiling the following conditions:

(1) $f_{I}\left(0_{\mathcal{L}^{I}}\right)=0_{\mathcal{L}^{I}}, f_{I}\left(1_{\mathcal{L}^{I}}\right)=1_{\mathcal{L}^{I}}$,

(2) $f_{I}(a) \leq_{L^{I}} f_{I}(b)$ whenever $a \leq_{L^{I}} b$,

such that

$$
\operatorname{card}_{I}(A)=\sum_{u \in \operatorname{supp}(A)} f_{I}(A(u))
$$

for each $A \in \mathcal{F}_{\mathcal{L}^{I}}^{F}(U)$.

We recall some definitions and properties that we will need later on. Valuation property: For each $A, B \in \mathcal{F}_{\mathcal{L}^{I}}^{F}(U)$,

$$
\operatorname{card}_{I}\left(A \cap_{\mathcal{T}} B\right)+\operatorname{card}_{I}\left(A \cup_{\mathcal{S}} B\right)=\operatorname{card}_{I}(A)+\operatorname{card}_{I}(B),
$$

for a t-norm $\mathcal{T}$ and a t-conorm $\mathcal{S}$ on $\mathcal{L}^{I}$.

Proposition 2.9 [11] The scalar cardinality induced by a cardinality pattern $f_{I}$ satisfies the valuation property for a t-norm $\mathcal{T}$ and a t-conorm $\mathcal{S}$ on $\mathcal{L}^{I}$ iff for each $a, b \in L^{I}$,

$$
f_{I}(\mathcal{T}(a, b))+f_{I}(\mathcal{S}(a, b))=f_{I}(a)+f_{I}(b) .
$$

\section{Definition 2.10 [11]}

- A scalar cardinality of interval-valued fuzzy sets card $_{I}$ is called representable if there exist scalar cardinalities of fuzzy sets card and $_{1}$ card $_{2}$ such that, for all $A \in \mathcal{F}_{\mathcal{L}^{I}}^{F}(U)$,

$$
\operatorname{card}_{I}(A)=\left[\operatorname{card}_{1}\left(A_{1}\right), \operatorname{card}_{2}\left(A_{2}\right)\right],
$$

where $A_{1}, A_{2} \in \mathcal{F}_{[0,1]}^{F}(U)$ are given by $A_{1}(u)=(A(u))_{1}$ and $A_{2}(u)=$ $(A(u))_{2}$, for all $u \in U$. We denote a representable cardinality by $\operatorname{card}_{I}^{r}$.

- A scalar cardinality of interval-valued fuzzy sets card $_{I}$ is called 1-semirepresentable if there exists a scalar cardinality of fuzzy sets card $_{1}$ such that, for all $A \in \mathcal{F}_{\mathcal{L}^{I}}^{F}(U)$,

$$
\left(\operatorname{card}_{I}(A)\right)_{1}=\operatorname{card}_{1}\left(A_{1}\right),
$$

where $A_{1} \in \mathcal{F}_{[0,1]}^{F}(U)$ is given by $A_{1}(u)=(A(u))_{1}$, for all $u \in U$. We denote a pseudo-representable cardinality by $\operatorname{card}_{I}^{s r 1}$. 
- A scalar cardinality of interval-valued fuzzy sets card $_{I}$ is called 2-semi-representable if there exists a scalar cardinality of fuzzy sets $\operatorname{card}_{2}$ such that, for all $A \in \mathcal{F}_{\mathcal{L}^{I}}^{F}(U)$,

$$
\left(\operatorname{card}_{I}(A)\right)_{2}=\operatorname{card}_{2}\left(A_{2}\right),
$$

where $A_{2} \in \mathcal{F}_{[0,1]}^{F}(U)$ is given by $A_{2}(u)=(A(u))_{2}$, for all $u \in U$. We denote a 2-semi-representable cardinality by $\operatorname{card}_{I}^{s r 2}$.

- A scalar cardinality pattern of interval-valued fuzzy sets $f_{I}$ is called representable if there exist scalar cardinality patterns of fuzzy sets $f_{1}$ and $f_{2}$ such that, for all $a \in L^{I}$,

$$
f_{I}(a)=\left[f_{1}\left(a_{1}\right), f_{2}\left(a_{2}\right)\right] .
$$

We denote a representable cardinality pattern by $f_{I}^{r}$.

- A scalar cardinality pattern of interval-valued fuzzy sets $f_{I}$ is called 1semi-representable if there exists a scalar cardinality pattern of fuzzy sets $f_{1}$ such that, for all $a \in L^{I}$,

$$
\left(f_{I}(a)\right)_{1}=f_{1}\left(a_{1}\right) .
$$

We denote a 1-semi-representable cardinality pattern by $f_{I}^{s r 1}$.

- A scalar cardinality pattern of interval-valued fuzzy sets $f_{I}$ is called 2semi-representable if there exists a scalar cardinality pattern of fuzzy sets $f_{2}$ such that, for all $a \in L^{I}$,

$$
\left(f_{I}(a)\right)_{2}=f_{2}\left(a_{2}\right) .
$$

We denote a 2-semi-representable cardinality pattern by $f_{I}^{s r 2}$.

A scalar cardinality which is 1- or 2-semi-representable will be called semirepresentable. Similarly, a scalar cardinality pattern which is 1- or 2-semi-representable will also be called semi-representable.

Theorem 2.11 [11] A scalar cardinality pattern $f_{I}$ is 1-semi-representable iff

$$
\left(f_{I}\left(\left[a_{1}, a_{2}\right]\right)\right)_{1}=\left(f_{I}\left(\left[a_{1}, a_{2}^{\prime}\right]\right)\right)_{1},
$$

for all $\left[a_{1}, a_{2}\right],\left[a_{1}, a_{2}^{\prime}\right] \in L^{I}$.

Theorem 2.12 [11] A scalar cardinality pattern $f_{I}$ is 2-semi-representable iff

$$
\left(f_{I}\left(\left[a_{1}, a_{2}\right]\right)\right)_{2}=\left(f_{I}\left(\left[a_{1}^{\prime}, a_{2}\right]\right)\right)_{2},
$$

for all $\left[a_{1}, a_{2}\right],\left[a_{1}^{\prime}, a_{2}\right] \in L^{I}$. 
Theorem 2.13 [11] A scalar cardinality pattern $f_{I}$ is representable iff

$$
\left(f_{I}\left(\left[a_{1}, a_{2}\right]\right)\right)_{1}=\left(f_{I}\left(\left[a_{1}, a_{2}^{\prime}\right]\right)\right)_{1} \text { and }\left(f_{I}\left(\left[a_{1}, a_{2}\right]\right)\right)_{2}=\left(f_{I}\left(\left[a_{1}^{\prime}, a_{2}\right]\right)\right)_{2},
$$

for all $\left[a_{1}, a_{2}\right],\left[a_{1}, a_{2}^{\prime}\right],\left[a_{1}^{\prime}, a_{2}\right] \in L^{I}$.

Theorem 2.14 [11] Let $\operatorname{card}_{I}$ be a scalar cardinality of interval-valued fuzzy sets and let $f_{I}$ be its associated cardinality pattern. Then $\operatorname{card}_{I}$ is 1-semirepresentable iff $f_{I}$ is 1-semi-representable. Furthermore, if $\left(\operatorname{card}_{I}(A)\right)_{1}=$ $\operatorname{card}_{1}\left(A_{1}\right)$ and $\left(f_{I}\right)_{1}=f_{1}$, then $f_{1}$ is the cardinality pattern associated to card ${ }_{1}$.

Theorem 2.15 [11] Let $\operatorname{card}_{I}$ be a scalar cardinality of interval-valued fuzzy sets and let $f_{I}$ be its associated cardinality pattern. Then $\operatorname{card}_{I}$ is 2-semirepresentable iff $f_{I}$ is 2-semi-representable. Furthermore, if $\left(\operatorname{card}_{I}(A)\right)_{2}=$ $\operatorname{card}_{2}\left(A_{2}\right)$ and $\left(f_{I}\right)_{2}=f_{2}$, then $f_{2}$ is the cardinality pattern associated to card ${ }_{2}$.

Theorem 2.16 [11] Let $\operatorname{card}_{I}$ be a scalar cardinality of interval-valued fuzzy sets and let $f_{I}$ be its associated cardinality pattern. Then $\operatorname{card}_{I}$ is representable iff $f_{I}$ is representable. Furthermore, if $\operatorname{card}_{I}=\left[\operatorname{card}_{1}, \operatorname{card}_{2}\right]$ and $f_{I}=\left[f_{1}, f_{2}\right]$, then $f_{1}$ and $f_{2}$ are the cardinality patterns associated to $\operatorname{card}_{1}$ and $\operatorname{card}_{2}$ respectively.

\section{The valuation property and the class $\mathcal{T}_{T, t}$}

Proposition 3.1 Let $t_{1} \in[0,1], t_{2} \in[0,1]$, and let $\mathcal{T}_{T, t_{1}}$ and $\mathcal{S}_{S, t_{2}}$ be a generalized pseudo-t-representable $t$-norm and $t$-conorm on $\mathcal{L}^{I}$ respectively. If $\left(f_{I}, \mathcal{T}_{T, t_{1}}\right.$, $\left.\mathcal{S}_{S, t_{2}}\right)$ satisfies the valuation property, then for all $a \in L^{I}$,

$$
f_{I}\left(\left[0, \max \left(T\left(t_{1}, a_{2}\right), a_{1}\right)\right]\right)+f_{I}\left(\left[\min \left(S\left(1-t_{2}, a_{1}\right), a_{2}\right), 1\right]\right)=f_{I}(a)+f_{I}([0,1]) .
$$

Proof. Assume that $\mathcal{T}_{T, t_{1}}$ and $\mathcal{S}_{S, t_{2}}$ are generalized pseudo-t-representable and $\left(f_{I}, \mathcal{T}_{T, t_{1}}, \mathcal{S}_{S, t_{2}}\right)$ satisfies the valuation property. Then for all $a \in L^{I}$ it holds that

$$
f_{I}\left(\mathcal{T}_{T, t_{1}}(a,[0,1])\right)+f_{I}\left(\mathcal{S}_{S, t_{2}}(a,[0,1])\right)=f_{I}(a)+f_{I}([0,1]),
$$

from which the result immediately follows.

By putting $a=\left[a_{1}, a_{1}\right] \in D, a=\left[a_{1}, 1\right]$ and $a=\left[0, a_{2}\right]$ respectively, we obtain the following corollaries.

Corollary 3.2 Let $t_{1} \in[0,1], t_{2} \in[0,1]$, and let $\mathcal{T}_{T, t_{1}}$ and $\mathcal{S}_{S, t_{2}}$ be a generalized pseudo-t-representable $t$-norm and $t$-conorm on $\mathcal{L}^{I}$ respectively. If $\left(f_{I}, \mathcal{T}_{T, t_{1}}\right.$, $\left.\mathcal{S}_{S, t_{2}}\right)$ satisfies the valuation property, then for all $a_{1} \in[0,1]$,

$$
f_{I}\left(\left[0, a_{1}\right]\right)+f_{I}\left(\left[a_{1}, 1\right]\right)=f_{I}\left(\left[a_{1}, a_{1}\right]\right)+f_{I}([0,1]) .
$$


Corollary 3.3 Let $_{1} \in[0,1], t_{2} \in[0,1]$, and let $\mathcal{T}_{T, t_{1}}$ and $\mathcal{S}_{S, t_{2}}$ be a generalized pseudo-t-representable $t$-norm and $t$-conorm on $\mathcal{L}^{I}$ respectively. If $\left(f_{I}, \mathcal{T}_{T, t_{1}}\right.$, $\left.\mathcal{S}_{S, t_{2}}\right)$ satisfies the valuation property, then for all $a_{1} \in[0,1]$,

$$
f_{I}\left(\left[0, \max \left(t_{1}, a_{1}\right)\right]\right)+f_{I}\left(\left[S\left(1-t_{2}, a_{1}\right), 1\right]\right)=f_{I}\left(\left[a_{1}, 1\right]\right)+f_{I}([0,1]) .
$$

Corollary 3.4 Let $t_{1} \in[0,1], t_{2} \in[0,1]$, and let $\mathcal{T}_{T, t_{1}}$ and $\mathcal{S}_{S, t_{2}}$ be a generalized pseudo-t-representable $t$-norm and t-conorm on $\mathcal{L}^{I}$ respectively. If $\left(f_{I}, \mathcal{T}_{T, t_{1}}\right.$, $\left.\mathcal{S}_{S, t_{2}}\right)$ satisfies the valuation property, then for all $a_{2} \in[0,1]$,

$$
f_{I}\left(\left[0, T\left(t_{1}, a_{2}\right)\right]\right)+f_{I}\left(\left[\min \left(1-t_{2}, a_{2}\right), 1\right]\right)=f_{I}\left(\left[0, a_{2}\right]\right)+f_{I}([0,1]) .
$$

By putting $a_{2}=1$ in the previous corollary we obtain the following.

Corollary 3.5 Let $t_{1} \in[0,1], t_{2} \in[0,1]$, and let $\mathcal{T}_{T, t_{1}}$ and $\mathcal{S}_{S, t_{2}}$ be a generalized pseudo-t-representable $t$-norm and $t$-conorm on $\mathcal{L}^{I}$ respectively. If $\left(f_{I}, \mathcal{T}_{T, t_{1}}\right.$, $\left.\mathcal{S}_{S, t_{2}}\right)$ satisfies the valuation property, then

$$
f_{I}\left(\left[0, t_{1}\right]\right)+f_{I}\left(\left[1-t_{2}, 1\right]\right)=f_{I}([0,1])+f_{I}([0,1]) .
$$

Corollary 3.6 Let $t_{1} \in[0,1], t_{2} \in[0,1]$, and let $\mathcal{T}_{T, t_{1}}$ and $\mathcal{S}_{S, t_{2}}$ be a generalized pseudo-t-representable $t$-norm and $t$-conorm on $\mathcal{L}^{I}$ respectively. Let $f_{I}$ be 1-semi-representable. If $\left(f_{I}, \mathcal{T}_{T, t_{1}}, \mathcal{S}_{S, t_{2}}\right)$ satisfies the valuation property, then $f_{1}\left(1-t_{2}\right)=0$.

Proof. Under the given conditions it follows from Corollary 3.5 that $f_{1}(0)+$ $f_{1}\left(1-t_{2}\right)=f_{1}(0)+f_{1}(0)$. Since $f_{1}(0)=0$, we immediately obtain that $f_{1}(1-$ $\left.t_{2}\right)=0$.

Corollary 3.7 Let $t_{1} \in[0,1], t_{2} \in[0,1]$, and let $\mathcal{T}_{T, t_{1}}$ and $\mathcal{S}_{S, t_{2}}$ be a generalized pseudo-t-representable $t$-norm and $t$-conorm on $\mathcal{L}^{I}$ respectively. Let $f_{I}$ be 2-semi-representable. If $\left(f_{I}, \mathcal{T}_{T, t_{1}}, \mathcal{S}_{S, t_{2}}\right)$ satisfies the valuation property, then $f_{2}\left(t_{1}\right)=1$.

Proof. Under the given conditions it follows from Corollary 3.5 that $f_{2}\left(t_{1}\right)+$ $f_{2}(1)=f_{2}(1)+f_{2}(1)$. Since $f_{2}(1)=1$, we immediately obtain that $f_{2}\left(t_{1}\right)=1$.

Theorem 3.8 Let $t_{1} \in[0,1], t_{2} \in[0,1]$, and let $\mathcal{T}_{T, t_{1}}$ and $\mathcal{S}_{S, t_{2}}$ be a generalized pseudo-t-representable $t$-norm and $t$-conorm on $\mathcal{L}^{I}$ respectively. If $\left(f_{I}, \mathcal{T}_{T, t_{1}}\right.$, $\left.\mathcal{S}_{S, t_{2}}\right)$ satisfies the valuation property, then

- for all $a \in L^{I}$ for which $a_{1} \leq T\left(t_{1}, a_{2}\right)$ and $a_{2} \geq S\left(1-t_{2}, a_{1}\right)$ it holds that

$$
f_{I}\left(\left[0, a_{2}\right]\right)+f_{I}\left(\left[a_{1}, 1\right]\right)=f_{I}(a)+f_{I}([0,1]) ;
$$


- for all $a \in L^{I}$ for which $a_{1} \geq T\left(t_{1}, a_{2}\right)$ and $a_{2} \leq S\left(1-t_{2}, a_{1}\right)$ it holds that

$$
f_{I}\left(\left[0, a_{1}\right]\right)+f_{I}\left(\left[a_{2}, 1\right]\right)=f_{I}(a)+f_{I}([0,1]) ;
$$

- for all $a \in L^{I}$ for which $a_{1} \geq T\left(t_{1}, a_{2}\right)$ and $a_{2} \geq S\left(1-t_{2}, a_{1}\right)$ it holds that

$$
f_{I}\left(\left[0, a_{1}\right]\right)+f_{I}\left(\left[a_{1}, 1\right]\right)=f_{I}(a)+f_{I}\left(\left[0, \max \left(t_{1}, a_{1}\right)\right]\right) ;
$$

- for all $a \in L^{I}$ for which $a_{1} \leq T\left(t_{1}, a_{2}\right)$ and $a_{2} \leq S\left(1-t_{2}, a_{1}\right)$ it holds that

$$
f_{I}\left(\left[a_{2}, 1\right]\right)+f_{I}\left(\left[0, a_{2}\right]\right)=f_{I}(a)+f_{I}\left(\left[\min \left(1-t_{2}, a_{2}\right), 1\right]\right) .
$$

Proof. Assume that $\mathcal{T}_{T, t_{1}}$ and $\mathcal{S}_{S, t_{2}}$ are generalized pseudo-t-representable and $\left(f_{I}, \mathcal{T}_{T, t_{1}}, \mathcal{S}_{S, t_{2}}\right)$ satisfies the valuation property.

- From Corollary 3.3 it follows for all $a_{1} \in[0,1]$ for which $a_{1} \leq t_{1}$, that

$$
f_{I}\left(\left[0, t_{1}\right]\right)+f_{I}\left(\left[S\left(1-t_{2}, a_{1}\right), 1\right]\right)=f_{I}\left(\left[a_{1}, 1\right]\right)+f_{I}([0,1]) .
$$

On the other hand, for all $a \in L^{I}$ satisfying $a_{1} \leq T\left(t_{1}, a_{2}\right)$ we obtain from Proposition 3.1 and Corollary 3.4 that

$$
\begin{aligned}
f_{I}\left(\left[0, T\left(t_{1}, a_{2}\right)\right]\right)+f_{I}\left(\left[\min \left(S\left(1-t_{2}, a_{1}\right), a_{2}\right), 1\right]\right) & =f_{I}(a)+f_{I}([0,1]), \\
f_{I}\left(\left[0, T\left(t_{1}, a_{2}\right)\right]\right)+f_{I}\left(\left[\min \left(1-t_{2}, a_{2}\right), 1\right]\right) & =f_{I}\left(\left[0, a_{2}\right]\right)+f_{I}([0,1]),
\end{aligned}
$$

from which it follows that

$$
f_{I}\left(\left[\min \left(S\left(1-t_{2}, a_{1}\right), a_{2}\right), 1\right]\right)+f_{I}\left(\left[0, a_{2}\right]\right)=f_{I}(a)+f_{I}\left(\left[\min \left(1-t_{2}, a_{2}\right), 1\right]\right) .
$$

If furthermore $a_{2} \geq S\left(1-t_{2}, a_{1}\right)$, then also $a_{2} \geq 1-t_{2}$, so we obtain

$$
f_{I}\left(\left[S\left(1-t_{2}, a_{1}\right), 1\right]\right)+f_{I}\left(\left[0, a_{2}\right]\right)=f_{I}(a)+f_{I}\left(\left[1-t_{2}, 1\right]\right) .
$$

From (6) and (7) it follows that

$$
f_{I}\left(\left[0, t_{1}\right]\right)+f_{I}(a)+f_{I}\left(\left[1-t_{2}, 1\right]\right)=f_{I}\left(\left[0, a_{2}\right]\right)+f_{I}\left(\left[a_{1}, 1\right]\right)+f_{I}([0,1]),
$$

for all $a \in L^{I}$ for which $a_{1} \leq T\left(t_{1}, a_{2}\right)$ and $a_{2} \geq S\left(1-t_{2}, a_{1}\right)$. Using Corollary 3.5 it follows that

$$
f_{I}([0,1])+f_{I}(a)=f_{I}\left(\left[0, a_{2}\right]\right)+f_{I}\left(\left[a_{1}, 1\right]\right),
$$

for all $a \in L^{I}$ for which $a_{1} \leq T\left(t_{1}, a_{2}\right)$ and $a_{2} \geq S\left(1-t_{2}, a_{1}\right)$.

- Let $a \in L^{I}$ with $a_{1} \geq T\left(t_{1}, a_{2}\right)$ and $a_{2} \leq S\left(1-t_{2}, a_{1}\right)$. Then from Proposition 3.1 it follows that

$$
f_{I}\left(\left[0, a_{1}\right]\right)+f_{I}\left(\left[a_{2}, 1\right]\right)=f_{I}(a)+f_{I}([0,1]) .
$$


- Let $a \in L^{I}$ such that $a_{1} \geq T\left(t_{1}, a_{2}\right)$ and $a_{2} \geq S\left(1-t_{2}, a_{1}\right)$. Then from Proposition 3.1 it follows that

$$
f_{I}\left(\left[0, a_{1}\right]\right)+f_{I}\left(\left[S\left(1-t_{2}, a_{1}\right), 1\right]\right)=f_{I}(a)+f_{I}([0,1]) .
$$

Combining this with Corollary 3.3, we obtain

$$
f_{I}\left(\left[0, a_{1}\right]\right)+f_{I}\left(\left[a_{1}, 1\right]\right)=f_{I}(a)+f_{I}\left(\left[0, \max \left(t_{1}, a_{1}\right)\right]\right) .
$$

- Let $a \in L^{I}$ such that $a_{1} \leq T\left(t_{1}, a_{2}\right)$ and $a_{2} \leq S\left(1-t_{2}, a_{1}\right)$. Then from Proposition 3.1 it follows that

$$
f_{I}\left(\left[0, T\left(t_{1}, a_{2}\right)\right]\right)+f_{I}\left(\left[a_{2}, 1\right]\right)=f_{I}(a)+f_{I}([0,1]) .
$$

Combining this with Corollary 3.4, we obtain

$$
f_{I}\left(\left[a_{2}, 1\right]\right)+f_{I}\left(\left[0, a_{2}\right]\right)=f_{I}(a)+f_{I}\left(\left[\min \left(1-t_{2}, a_{2}\right), 1\right]\right) .
$$

Theorem 3.8 shows that if $\left(f_{I}, \mathcal{T}_{T, t_{1}}, \mathcal{S}_{S, t_{2}}\right)$ satisfies the valuation property, with $\mathcal{T}_{T, t_{1}}$ and $\mathcal{S}_{S, t_{2}}$ generalized pseudo-t-representable, then $f_{I}$ is completely determined by its action on $\left\{\left[0, x_{2}\right] \mid x_{2} \in[0,1]\right\} \cup\left\{\left[x_{1}, 1\right] \mid x_{1} \in[0,1]\right\}, T$ and $S$.

Corollary 3.9 Let $t_{1} \in[0,1], t_{2} \in[0,1]$, and let $\mathcal{T}_{T, t_{1}}$ and $\mathcal{S}_{S, t_{2}}$ be a generalized pseudo-t-representable $t$-norm and $t$-conorm on $\mathcal{L}^{I}$ respectively. If $\left(f_{I}, \mathcal{T}_{T, t_{1}}\right.$, $\left.\mathcal{S}_{S, t_{2}}\right)$ satisfies the valuation property, then

$$
f_{I}\left(\left[t_{1}, 1-t_{2}\right]\right)=f_{I}([0,1]) .
$$

Proof. Let $a=\left[t_{1}, 1-t_{2}\right]$, then obviously $a_{1} \geq T\left(t_{1}, a_{2}\right)$ and $a_{2} \leq S\left(1-t_{2}, a_{1}\right)$, so from Theorem 3.8 it follows that

$$
f_{I}\left(\left[0, t_{1}\right]\right)+f_{I}\left(\left[1-t_{2}, 1\right]\right)=f_{I}\left(\left[t_{1}, 1-t_{2}\right]\right)+f_{I}([0,1]),
$$

Using Corollary 3.5 we obtain the desired result.

\section{The valuation property and the class $\mathcal{T}_{T}$}

Lemma 4.1 Let $\mathcal{T}_{T}$ and $\mathcal{S}_{S}$ be a pseudo-t-representable $t$-norm and $t$-conorm of the first kind on $\mathcal{L}^{I}$ respectively. If $\left(f_{I}, \mathcal{T}_{T}, \mathcal{S}_{S}\right)$ satisfies the valuation property, then, for all $a \in L^{I}$,

$$
f_{I}(a)=f_{I}\left(\left[0, a_{1}\right]\right)+f_{I}\left(\left[0, a_{2}\right]\right) .
$$


Proof. Let $b=[0,1]$, then from (2) it follows that, for all $a \in L^{I}$,

$$
f_{I}\left(\left[0, a_{1}\right]\right)+f_{I}\left(\left[a_{2}, 1\right]\right)=f_{I}(a)+f_{I}([0,1]) .
$$

Let also $a=[0,1]$, then we obtain that $[1,1]=f_{I}([0,0])+f_{I}([1,1])=f_{I}([0,1])+$ $f_{I}([0,1])$. Hence $f_{I}([0,1])=\left[\frac{1}{2}, \frac{1}{2}\right]$.

Let now $a=\left[a_{2}, 1\right]$, then from (8) it follows that

$$
f_{I}\left(\left[0, a_{2}\right]\right)+f_{I}([1,1])=f_{I}\left(\left[a_{2}, 1\right]\right)+f_{I}([0,1]) .
$$

Using the fact that $f_{I}([1,1])=f_{I}([0,1])+f_{I}([0,1])$, we obtain

$$
f_{I}\left(\left[a_{2}, 1\right]\right)=f_{I}\left(\left[0, a_{2}\right]\right)+f_{I}([0,1]) .
$$

Using (9) in (8), we obtain that, for all $a \in L^{I}$,

$$
f_{I}\left(\left[0, a_{1}\right]\right)+f_{I}\left(\left[0, a_{2}\right]\right)+f_{I}([0,1])=f_{I}(a)+f_{I}([0,1]) .
$$

Hence we obtain what we had to prove.

Lemma 4.2 Let $\mathcal{T}_{T}$ and $\mathcal{S}_{S}$ be a pseudo-t-representable $t$-norm and $t$-conorm of the first kind on $\mathcal{L}^{I}$ respectively. If $\left(f_{I}, \mathcal{T}_{T}, \mathcal{S}_{S}\right)$ satisfies the valuation property, then, for all $a, b \in[0,1]$,

$$
\begin{aligned}
& f_{I}([0, T(a, b)])=f_{I}([0, \min (a, b)]), \\
& f_{I}([0, S(a, b)])=f_{I}([0, \max (a, b)]) .
\end{aligned}
$$

Proof. Let $x_{2}, y_{2} \in[0,1]$ be arbitrary, then $\mathcal{T}_{T}\left(\left[0, x_{2}\right],\left[0, y_{2}\right]\right)=[0,0]$ and $\mathcal{S}_{S}\left(\left[0, x_{2}\right],\left[0, y_{2}\right]\right)=\left[\min \left(x_{2}, y_{2}\right), S\left(x_{2}, y_{2}\right)\right]$. So, from (2) it follows that

$$
f_{I}([0,0])+f_{I}\left(\left[\min \left(x_{2}, y_{2}\right), S\left(x_{2}, y_{2}\right)\right]\right)=f_{I}\left(\left[0, x_{2}\right]\right)+f_{I}\left(\left[0, y_{2}\right]\right) .
$$

Using Lemma 4.1 we obtain

$$
f_{I}\left(\left[0, \min \left(x_{2}, y_{2}\right)\right]\right)+f_{I}\left(\left[0, S\left(x_{2}, y_{2}\right)\right]\right)=f_{I}\left(\left[0, x_{2}\right]\right)+f_{I}\left(\left[0, y_{2}\right]\right) .
$$

If $x_{2} \leq y_{2}$, then from (10) it follows that

$$
f_{I}\left(\left[0, x_{2}\right]\right)+f_{I}\left(\left[0, S\left(x_{2}, y_{2}\right)\right]\right)=f_{I}\left(\left[0, x_{2}\right]\right)+f_{I}\left(\left[0, y_{2}\right]\right),
$$

or, equivalently,

$$
f_{I}\left(\left[0, S\left(x_{2}, y_{2}\right)\right]\right)=f_{I}\left(\left[0, y_{2}\right]\right) .
$$

Similarly, from $x_{2} \geq y_{2}$ it follows that $f_{I}\left(\left[0, S\left(x_{2}, y_{2}\right)\right]\right)=f_{I}\left(\left[0, x_{2}\right]\right)$. Hence, for all $x_{2}, y_{2} \in[0,1]$,

$$
f_{I}\left(\left[0, S\left(x_{2}, y_{2}\right)\right]\right)=f_{I}\left(\left[0, \max \left(x_{2}, y_{2}\right)\right]\right) .
$$


Let us now choose arbitrarily $x_{1}, y_{1} \in[0,1]$, then $\mathcal{T}_{T}\left(\left[x_{1}, x_{1}\right],\left[y_{1}, y_{1}\right]\right)=$ $\left[T\left(x_{1}, y_{1}\right), T\left(x_{1}, y_{1}\right)\right]$ and $\mathcal{S}_{S}\left(\left[x_{1}, x_{1}\right],\left[y_{1}, y_{1}\right]\right)=\left[S\left(x_{1}, y_{1}\right), S\left(x_{1}, y_{1}\right)\right]$. So, from (2) it follows that

$f_{I}\left(\left[T\left(x_{1}, y_{1}\right), T\left(x_{1}, y_{1}\right)\right]\right)+f_{I}\left(\left[S\left(x_{1}, y_{1}\right), S\left(x_{1}, y_{1}\right)\right]\right)=f_{I}\left(\left[x_{1}, x_{1}\right]\right)+f_{I}\left(\left[y_{1}, y_{1}\right]\right)$.

Note that for all $x, y \in L^{I}$, from $x+x=y+y$ it follows that $2 x_{1}=2 y_{1}$ and $2 x_{2}=2 y_{2}$, so $x=y$. Thus, using Lemma 4.1, we obtain

$$
f_{I}\left(\left[0, T\left(x_{1}, y_{1}\right)\right]\right)+f_{I}\left(\left[0, S\left(x_{1}, y_{1}\right)\right]\right)=f_{I}\left(\left[0, x_{1}\right]\right)+f_{I}\left(\left[0, y_{1}\right]\right) .
$$

Taking into account (11), it follows that

$$
f_{I}\left(\left[0, T\left(x_{1}, y_{1}\right)\right]\right)+f_{I}\left(\left[0, \max \left(x_{1}, y_{1}\right)\right]\right)=f_{I}\left(\left[0, x_{1}\right]\right)+f_{I}\left(\left[0, y_{1}\right]\right) .
$$

If $x_{1} \leq y_{1}$, then from (12) it follows that

$$
f_{I}\left(\left[0, T\left(x_{1}, y_{1}\right)\right]\right)+f_{I}\left(\left[0, y_{1}\right]\right)=f_{I}\left(\left[0, x_{1}\right]\right)+f_{I}\left(\left[0, y_{1}\right]\right),
$$

or, equivalently, $f_{I}\left(\left[0, T\left(x_{1}, y_{1}\right)\right]\right)=f_{I}\left(\left[0, x_{1}\right]\right)$. Similarly, if $x_{1} \geq y_{1}$, then $f_{I}\left(\left[0, T\left(x_{1}, y_{1}\right)\right]\right)=f_{I}\left(\left[0, y_{1}\right]\right)$. Hence, for all $x_{1}, y_{1} \in[0,1]$,

$$
f_{I}\left(\left[0, T\left(x_{1}, y_{1}\right)\right]\right)=f_{I}\left(\left[0, \min \left(x_{1}, y_{1}\right)\right]\right) .
$$

Theorem 4.3 Let $\mathcal{T}_{T}$ and $\mathcal{S}_{S}$ be a pseudo-t-representable $t$-norm and $t$-conorm of the first kind on $\mathcal{L}^{I}$ respectively. Then $\left(f_{I}, \mathcal{T}_{T}, \mathcal{S}_{S}\right)$ satisfies the valuation property iff

(i) for all $a \in L^{I}$,

$$
f_{I}(a)=f_{I}\left(\left[0, a_{1}\right]\right)+f_{I}\left(\left[0, a_{2}\right]\right)
$$

(ii) for all $a, b \in[0,1]$,

$$
\begin{aligned}
& f_{I}([0, T(a, b)])=f_{I}([0, \min (a, b)]), \\
& f_{I}([0, S(a, b)])=f_{I}([0, \max (a, b)]) .
\end{aligned}
$$

Proof. From Lemmas 4.1 and 4.2 it follows that if $\left(f_{I}, \mathcal{T}_{T}, \mathcal{S}_{S}\right)$ satisfies the valuation property, then (i) and (ii) hold.

Assume conversely that (i) and (ii) hold and let $x, y \in L^{I}$ be arbitrary. Then, using (i), (2) is equivalent to

$$
\begin{aligned}
& f_{I}\left(\left[0, T\left(x_{1}, y_{1}\right)\right]\right)+f_{I}\left(\left[0, \max \left(T\left(x_{1}, y_{2}\right), T\left(x_{2}, y_{1}\right)\right)\right]\right) \\
& +f_{I}\left(\left[0, \min \left(S\left(x_{1}, y_{2}\right), S\left(x_{2}, y_{1}\right)\right)\right]\right)+f_{I}\left(\left[0, S\left(x_{2}, y_{2}\right)\right]\right) \\
& =f_{I}\left(\left[0, x_{1}\right]\right)+f_{I}\left(\left[0, x_{2}\right]\right)+f_{I}\left(\left[0, y_{1}\right]\right)+f_{I}\left(\left[0, y_{2}\right]\right)
\end{aligned}
$$


or, using (ii), equivalent to

$$
\begin{aligned}
& f_{I}\left(\left[0, \min \left(x_{1}, y_{1}\right)\right]\right)+f_{I}\left(\left[0, \max \left(T\left(x_{1}, y_{2}\right), T\left(x_{2}, y_{1}\right)\right)\right]\right) \\
& +f_{I}\left(\left[0, \min \left(S\left(x_{1}, y_{2}\right), S\left(x_{2}, y_{1}\right)\right)\right]\right)+f_{I}\left(\left[0, \max \left(x_{2}, y_{2}\right)\right]\right) \\
& =f_{I}\left(\left[0, x_{1}\right]\right)+f_{I}\left(\left[0, x_{2}\right]\right)+f_{I}\left(\left[0, y_{1}\right]\right)+f_{I}\left(\left[0, y_{2}\right]\right),
\end{aligned}
$$

We consider the following cases.

- If $x_{1} \leq y_{1}$ and $x_{2} \geq y_{2}$, then (2) is equivalent to

$$
\begin{aligned}
& f_{I}\left(\left[0, x_{1}\right]\right)+f_{I}\left(\left[0, T\left(x_{2}, y_{1}\right)\right]\right)+f_{I}\left(\left[0, S\left(x_{1}, y_{2}\right)\right]\right)+f_{I}\left(\left[0, x_{2}\right]\right) \\
& =f_{I}\left(\left[0, x_{1}\right]\right)+f_{I}\left(\left[0, x_{2}\right]\right)+f_{I}\left(\left[0, y_{1}\right]\right)+f_{I}\left(\left[0, y_{2}\right]\right),
\end{aligned}
$$

or, equivalently,

$$
f_{I}\left(\left[0, \min \left(x_{2}, y_{1}\right)\right]\right)+f_{I}\left(\left[0, \max \left(x_{1}, y_{2}\right)\right]\right)=f_{I}\left(\left[0, y_{1}\right]\right)+f_{I}\left(\left[0, y_{2}\right]\right),
$$

which holds, since $y_{1} \leq y_{2} \leq x_{2}$ and $y_{2} \geq y_{1} \geq x_{1}$.

- If $x_{1} \leq y_{1}$ and $x_{2} \leq y_{2}$, then (2) is equivalent to

$$
\begin{aligned}
& f_{I}\left(\left[0, \max \left(T\left(x_{1}, y_{2}\right), T\left(x_{2}, y_{1}\right)\right)\right]\right)+f_{I}\left(\left[0, \min \left(S\left(x_{1}, y_{2}\right), S\left(x_{2}, y_{1}\right)\right)\right]\right) \\
& =f_{I}\left(\left[0, x_{2}\right]\right)+f_{I}\left(\left[0, y_{1}\right]\right) .
\end{aligned}
$$

Let

$$
\alpha=f_{I}\left(\left[0, \max \left(T\left(x_{1}, y_{2}\right), T\left(x_{2}, y_{1}\right)\right)\right]\right)+f_{I}\left(\left[0, \min \left(S\left(x_{1}, y_{2}\right), S\left(x_{2}, y_{1}\right)\right)\right]\right) .
$$

Since $T, S$, min, max and $f_{I}$ are increasing and $y_{2} \leq 1$, we obtain that

$$
\begin{aligned}
\alpha & \leq_{\bar{L}^{I}} f_{I}\left(\left[0, \max \left(x_{1}, T\left(x_{2}, y_{1}\right)\right)\right]\right)+f_{I}\left(\left[0, S\left(x_{2}, y_{1}\right)\right]\right) \\
& \leq_{\bar{L}^{I}} f_{I}\left(\left[0, \min \left(x_{2}, y_{1}\right)\right]\right)+f_{I}\left(\left[0, \max \left(x_{2}, y_{1}\right)\right]\right),
\end{aligned}
$$

using the fact that $x_{1} \leq \min \left(x_{2}, y_{1}\right), T\left(x_{2}, y_{1}\right) \leq \min \left(x_{2}, y_{1}\right)$ and (ii). It is easily seen that

$$
f_{I}\left(\left[0, \min \left(x_{2}, y_{1}\right)\right]\right)+f_{I}\left(\left[0, \max \left(x_{2}, y_{1}\right)\right]\right)=f_{I}\left(\left[0, x_{2}\right]\right)+f_{I}\left(\left[0, y_{1}\right]\right) .
$$

On the other hand, if $x_{2} \geq y_{1}$, then, since $T, S$, min, max and $f_{I}$ are increasing and $x_{2} \leq y_{2}$, we obtain that

$$
\begin{aligned}
\alpha \geq \geq_{\bar{L}^{I}} & f_{I}\left(\left[0, \max \left(T\left(x_{1}, x_{2}\right), T\left(x_{2}, y_{1}\right)\right)\right]\right) \\
& +f_{I}\left(\left[0, \min \left(S\left(x_{1}, x_{2}\right), S\left(x_{2}, y_{1}\right)\right)\right]\right) \\
= & f_{I}\left(\left[0, T\left(x_{2}, y_{1}\right)\right]\right)+f_{I}\left(\left[0, S\left(x_{1}, x_{2}\right)\right]\right) \\
= & f_{I}\left(\left[0, \min \left(x_{2}, y_{1}\right)\right]\right)+f_{I}\left(\left[0, \max \left(x_{1}, x_{2}\right)\right]\right) \\
= & f_{I}\left(\left[0, y_{1}\right]\right)+f_{I}\left(\left[0, x_{2}\right]\right),
\end{aligned}
$$


using (ii). From (13), (14) and (15) it follows that $\alpha=f_{I}\left(\left[0, x_{2}\right]\right)+$ $f_{I}\left(\left[0, y_{1}\right]\right)$.

If $x_{2} \leq y_{1}$, since $T, S$, min, $\max$ and $f_{I}$ are increasing, $S\left(x_{1}, y_{2}\right) \geq y_{2} \geq y_{1}$, $S\left(x_{2}, y_{1}\right) \geq y_{1}$ and $x_{2} \leq y_{2}$, we obtain that

$$
\begin{aligned}
\alpha \geq_{\bar{L}^{I}} & f_{I}\left(\left[0, \max \left(T\left(x_{1}, x_{2}\right), T\left(x_{2}, y_{1}\right)\right)\right]\right) \\
& \quad+f_{I}\left(\left[0, \min \left(S\left(x_{1}, y_{2}\right), S\left(x_{2}, y_{1}\right)\right)\right]\right) \\
\geq_{\bar{L}^{I}} & f_{I}\left(\left[0, T\left(x_{2}, y_{1}\right)\right]\right)+f_{I}\left(\left[0, y_{1}\right]\right) \\
= & f_{I}\left(\left[0, \min \left(x_{2}, y_{1}\right)\right]\right)+f_{I}\left(\left[0, y_{1}\right]\right) \\
= & f_{I}\left(\left[0, x_{2}\right]\right)+f_{I}\left(\left[0, y_{1}\right]\right),
\end{aligned}
$$

using (ii). From (13), (14) and (16) it follows that $\alpha=f_{I}\left(\left[0, x_{2}\right]\right)+$ $f_{I}\left(\left[0, y_{1}\right]\right)$.

The other cases are analogous.

Corollary 4.4 Let $\mathcal{T}_{T}$ and $\mathcal{S}_{S}$ be a pseudo-t-representable t-norm and t-conorm of the first kind on $\mathcal{L}^{I}$ respectively. Let $f_{I}([0,]$.$) be strictly increasing. Then$ $\left(f_{I}, \mathcal{S}_{S}, \mathcal{T}_{T}\right)$ satisfies the valuation property iff $f_{I}$ satisfies Theorem $4.3(i), S=$ $\max$ and $T=\min$.

Proof. Assume that $\mathcal{T}_{T}$ and $\mathcal{S}_{S}$ are a pseudo-t-representable t-norm and t-conorm of the first kind on $\mathcal{L}^{I}$ respectively, $f_{I}([0,]$.$) is strictly increasing$ and $\left(f_{I}, \mathcal{S}_{S}, \mathcal{T}_{T}\right)$ satisfies the valuation property. Then $f_{I}([0, S(a, b)])=f_{I}([0$, $\max (a, b)])$ and $f_{I}([0, T(a, b)])=f_{I}([0, \min (a, b)])$. Hence $S(a, b)=\max (a, b)$ and $T(a, b)=\min (a, b)$.

The converse part of the statement is obvious.

Corollary 4.5 Let $\mathcal{T}_{T}$ and $\mathcal{S}_{S}$ be a pseudo-t-representable $t$-norm and $t$-conorm of the first kind on $\mathcal{L}^{I}$ respectively. Then there does not exist a 1-semi-representable scalar cardinality pattern $f_{I}$ such that $\left(f_{I}, \mathcal{T}_{T}, \mathcal{S}_{S}\right)$ satisfies the valuation property.

Proof. Assume that $\left(f_{I}, \mathcal{S}_{S}, \mathcal{T}_{T}\right)$ satisfies the valuation property and $f_{I}$ is 1semi-representable. From Lemma 4.1 it follows that $f_{I}([0,1])=\left[\frac{1}{2}, \frac{1}{2}\right]$. From Theorem 2.11 we obtain $\left(f_{I}([0,1])\right)_{1}=0$, which is a contradiction.

Corollary 4.6 Let $\mathcal{T}_{T}$ and $\mathcal{S}_{S}$ be a pseudo-t-representable $t$-norm and $t$-conorm of the first kind on $\mathcal{L}^{I}$ respectively. Then there does not exist a 2-semi-representable scalar cardinality pattern $f_{I}$ such that $\left(f_{I}, \mathcal{T}_{T}, \mathcal{S}_{S}\right)$ satisfies the valuation property. 
Proof. Assume that $\left(f_{I}, \mathcal{S}_{S}, \mathcal{T}_{T}\right)$ satisfies the valuation property and $f_{I}$ is 2semi-representable. From Lemma 4.1 it follows that $f_{I}([0,1])=\left[\frac{1}{2}, \frac{1}{2}\right]$. From Theorem 2.12 we obtain $\left(f_{I}([0,1])\right)_{2}=1$, which is a contradiction.

Corollary 4.7 Let $\mathcal{T}_{T}$ and $\mathcal{S}_{S}$ be a pseudo-t-representable t-norm and t-conorm of the first kind on $\mathcal{L}^{I}$ respectively. Then there does not exist a representable scalar cardinality pattern $f_{I}$ such that $\left(f_{I}, \mathcal{T}_{T}, \mathcal{S}_{S}\right)$ satisfies the valuation property.

Note that from Theorem 4.3 it follows that in the case that $\mathcal{T}$ and $\mathcal{S}$ are pseudo-t-representable of the first kind, if $\left(f_{I}, \mathcal{T}, \mathcal{S}\right)$ satisfies the valuation property, then $f_{I}$ is completely determined by the value of $f_{I}([0, a])$, for all $a \in[0,1]$. In the case that the representant of one of $\mathcal{T}$ and $\mathcal{S}$ is Archimedean, we can find even stronger results. First we prove some lemmas. Let us remind the reader that $T^{*}\left(S^{*}\right)$ denotes the dual t-conorm (t-norm) to a t-norm $T$ (t-conorm $S$ ) with respect to the standard negation $\mathcal{N}_{s}$, respectively.

Lemma 4.8 Let $\mathcal{T}_{T}$ and $\mathcal{S}_{S}$ be a pseudo-t-representable t-norm and t-conorm of the first kind on $\mathcal{L}^{I}$ respectively. If at least one of $T$ and $S^{*}$ is Archimedean and $\left(f_{I}, \mathcal{T}_{T}, \mathcal{S}_{S}\right)$ satisfies the valuation property, then $f_{I}([0, a])=f_{I}([0, b])$, for all $a, b \in] 0,1[$.

Proof. Assume first that $T$ is Archimedean. Let us choose arbitrarily $a, b \in$ ]0,1[ and assume $a \geq b$ (the case $a \leq b$ is shown similarly). From Theorem 4.3 it follows that $f_{I}\left(\left[0, a_{T}^{(2)}\right]\right)=f_{I}([0, T(a, a)])=f_{I}([0, \min (a, a)])=f_{I}([0, a])$. Recursively, using Theorem 4.3, we find for all $n>2$ that

$$
\begin{aligned}
f_{I}\left(\left[0, a_{T}^{(n)}\right]\right) & =f_{I}\left(\left[0, T\left(a, a_{T}^{(n-1)}\right)\right]\right) \\
& =f_{I}\left(\left[0, \min \left(a, a_{T}^{(n-1)}\right)\right]\right) \\
& =f_{I}\left(\left[0, a_{T}^{(n-1)}\right]\right) \\
& =f_{I}([0, a]) .
\end{aligned}
$$

Since $T$ is Archimedean, there exists an $n \in \mathbb{N} \backslash\{0\}$ such that $a_{T}^{(n)}<b$. Hence, since $f_{I}$ is increasing,

$$
f_{I}([0, a])=f_{I}\left(\left[0, a_{T}^{(n)}\right]\right) \leq_{L^{I}} f_{I}([0, b]) \leq_{L^{I}} f_{I}([0, a]) .
$$

Assume now that $S^{*}$ is Archimedean. We have for all $x \in[0,1]$ that $x_{S}^{(2)}=$ $S(x, x)=1-S^{*}(1-x, 1-x)=1-(1-x)_{S^{*}}^{(2)}$. We find recursively for all $n>2$, that

$$
\begin{aligned}
x_{S}^{(n)} & =S\left(x, x_{S}^{(n-1)}\right) \\
& =1-S^{*}\left(1-x, 1-x_{S}^{(n-1)}\right) \\
& =1-S^{*}\left(1-x,(1-x)_{S^{*}}^{(n-1)}\right) \\
& =1-(1-x)_{S^{*}}^{(n)} .
\end{aligned}
$$


Since $S^{*}$ is Archimedean, for all $\left.x, y \in\right] 0,1[$, there exists an $n \in \mathbb{N} \backslash\{0\}$ such that $(1-x)_{S^{*}}^{(n)}<1-y$, so $x_{S}^{(n)}=1-(1-x)_{S^{*}}^{(n)}>y$.

Let now arbitrarily $a, b \in] 0,1[$ and assume $a \leq b$ (the case $a \geq b$ is shown similarly). From Theorem 4.3 it follows that $f_{I}\left(\left[0, a_{S}^{(2)}\right]\right)=f_{I}([0, S(a, a)])=$ $f_{I}([0, \max (a, a)])=f_{I}([0, a])$. Similarly as above for $T$, we find recursively that $f_{I}\left(\left[0, a_{S}^{(n)}\right]\right)=f_{I}([0, a])$, for all $n \in \mathbb{N} \backslash\{0\}$. Since $S^{*}$ is Archimedean, there exists an $n \in \mathbb{N} \backslash\{0\}$ such that $a_{S}^{(n)}>b$. Hence, since $f_{I}$ is increasing,

$$
f_{I}([0, a])=f_{I}\left(\left[0, a_{S}^{(n)}\right]\right) \geq_{L^{I}} f_{I}([0, b]) \geq_{L^{I}} f_{I}([0, a]) .
$$

Lemma 4.9 Let $\mathcal{T}_{T}$ and $\mathcal{S}_{S}$ be a pseudo-t-representable t-norm and $t$-conorm of the first kind on $\mathcal{L}^{I}$ respectively. If at least one of $T$ and $S^{*}$ is Archimedean and $\left(f_{I}, \mathcal{T}_{T}, \mathcal{S}_{S}\right)$ satisfies the valuation property, then there exists an $\alpha \in\left\{x \mid x \in L^{I}\right.$ and $\left.x_{2} \leq \frac{1}{2}\right\}$ such that, for all $a \in L^{I}$,

$$
f_{I}(a)= \begin{cases}0_{\mathcal{L}^{I}}, & \text { if } a=0_{\mathcal{L}^{I}}, \\ {\left[\frac{1}{2}, \frac{1}{2}\right],} & \text { if } a=[0,1] \\ 1_{\mathcal{L}^{I}}, & \text { if } a=1_{\mathcal{L}^{I}}, \\ \alpha, & \text { if } \left.a_{1}=0 \text { and } a_{2} \in\right] 0,1[, \\ {\left[\frac{1}{2}+\alpha_{1}, \frac{1}{2}+\alpha_{2}\right],} & \text { if } \left.a_{1} \in\right] 0,1\left[\text { and } a_{2}=1,\right. \\ {\left[2 \alpha_{1}, 2 \alpha_{2}\right],} & \text { else. }\end{cases}
$$

Proof. From the definition of scalar cardinality pattern and from the proof of Lemma 4.1 it follows that $f_{I}\left(0_{\mathcal{L}^{I}}\right)=0_{\mathcal{L}^{I}}, f_{I}\left(1_{\mathcal{L}^{I}}\right)=1_{\mathcal{L}^{I}}$ and $f_{I}([0,1])=\left[\frac{1}{2}, \frac{1}{2}\right]$.

From Lemma 4.8 it follows that there exists an $\alpha \in L^{I}$ such that $f_{I}([0, a])=$ $\alpha$, for all $a \in] 0,1\left[\right.$. Since $f_{I}$ is increasing, $f_{I}([0, a]) \leq_{L^{I}} f_{I}([0,1])$, so $\alpha_{2} \leq \frac{1}{2}$. Hence, for all $a \in[0,1]$,

$$
f_{I}([0, a])= \begin{cases}0_{\mathcal{L}^{I}}, & \text { if } a=0, \\ {\left[\frac{1}{2}, \frac{1}{2}\right],} & \text { if } a=1, \\ \alpha, & \text { else. }\end{cases}
$$

Using Theorem 4.3(i) it is easy to see that $f_{I}(a)$ is given by (17), for all $a \in L^{I}$.

Theorem 4.10 Let $\mathcal{T}_{T}$ and $\mathcal{S}_{S}$ be a pseudo-t-representable t-norm and t-conorm of the first kind on $\mathcal{L}^{I}$ respectively. If at least one of $T$ and $S^{*}$ is Archimedean and at least one of them does not have zero-divisors, then $\left(f_{I}, \mathcal{T}_{T}\right.$, $\left.\mathcal{S}_{S}\right)$ satisfies the valuation property iff there exists an $\alpha \in\left\{x \mid x \in L^{I}\right.$ and $x_{2} \leq \frac{1}{2}$ \} such that $f_{I}(a)$ is given by $(17)$, for all $a \in L^{I}$.

Moreover, if $T$ has zero-divisors, then $\alpha=0_{\mathcal{L}^{I}}$, and if $S^{*}$ has zero-divisors, then $\alpha=\left[\frac{1}{2}, \frac{1}{2}\right]$. 
Proof. Assume first that $\left(f_{I}, \mathcal{T}_{T}, \mathcal{S}_{S}\right)$ satisfies the valuation property. From Lemma 4.9 it follows that $f_{I}(a)$ is given by (17), for all $a \in L^{I}$.

Assume conversely that there exists an $\alpha \in L^{I}$ such that $\alpha_{2} \leq \frac{1}{2}$ and such that $f_{I}(a)$ is given by (17), for all $a \in L^{I}$. It is easy to see that $f_{I}$ is a scalar cardinality pattern and that $f_{I}([0, a])$ is given by (18), for all $a \in[0,1]$.

We prove that $\left(f_{I}, \mathcal{T}_{T}, \mathcal{S}_{S}\right)$ satisfies the valuation property. A straightforward calculation shows that $f_{I}(a)=f_{I}\left(\left[0, a_{1}\right]\right)+f_{I}\left(\left[0, a_{2}\right]\right)$, for all $a \in L^{I}$. Let now $a, b \in[0,1]$ such that $a \leq b$ (the case $a \geq b$ is analogous). If $a=0$, then $f_{I}([0, T(a, b)])=f_{I}([0,0])=f_{I}([0, \min (a, b)])$. If $b=1$, then $f_{I}([0, T(a, b)])=$ $f_{I}([0, a])=f_{I}([0, \min (a, b)])$. Similarly, if $a=0$ or $b=1$, then $f_{I}([0, S(a, b)])=$ $f_{I}([0, \max (a, b)])$. Assume now that $0<a \leq b<1$. Then we have the following cases.

- Neither $T$ or $S^{*}$ has zero-divisors: then $0<T(a, b)<1$ and

$$
\begin{aligned}
& a, b \in] 0,1[ \\
& \Longrightarrow 1-a, 1-b \in] 0,1[ \\
& \Longrightarrow 0<S^{*}(1-a, 1-b)<1 \\
& \Longrightarrow 0<S(a, b)<1 .
\end{aligned}
$$

Hence $f_{I}([0, T(a, b)])=\alpha=f_{I}([0, \min (a, b)])$ and $f_{I}([0, S(a, b)])=\alpha=$ $f_{I}([0, \max (a, b)])$.

- $T$ has zero-divisors: then from the assumptions it follows that $S^{*}$ does not have zero-divisors. So, similarly as in the previous case we find that $f_{I}([0, S(a, b)])=\alpha=f_{I}([0, \max (a, b)])$. Let $\left.a^{\prime}, b^{\prime} \in\right] 0,1[$ be such that $T\left(a^{\prime}, b^{\prime}\right)=0$. Then $f_{I}\left(\left[0, T\left(a^{\prime}, b^{\prime}\right)\right]\right)=f_{I}([0,0])=0_{\mathcal{L}^{I}}$, but on the other hand $f_{I}\left(\left[0, \min \left(a^{\prime}, b^{\prime}\right)\right]\right)=\alpha$. Thus, if $f_{I}\left(\left[0, T\left(a^{\prime}, b^{\prime}\right)\right]\right)=f_{I}([0$, $\left.\left.\min \left(a^{\prime}, b^{\prime}\right)\right]\right)$, then $\alpha=0_{\mathcal{L}^{I}}$. Conversely, if $\alpha=0_{\mathcal{L}^{I}}$, then for all $\left.a, b \in\right] 0,1[$, $T(a, b)<1$, so $f_{I}([0, T(a, b)])=0_{\mathcal{L}^{I}}=f_{I}([0, \min (a, b)])$.

- $S^{*}$ has zero-divisors: similarly as in the previous case we find that $T$ does not have zero-divisors, $f_{I}([0, T(a, b)])=\alpha=f_{I}([0, \min (a, b)])$ and $f_{I}([0, S(a, b)])=f_{I}([0, \max (a, b)])$ iff $\alpha=\left[\frac{1}{2}, \frac{1}{2}\right]$.

From Theorem 4.3 it follows that, under the mentioned restrictions for $\alpha$, $\left(f_{I}, \mathcal{T}_{T}, \mathcal{S}_{S}\right)$ satisfies the valuation property.

Theorem 4.11 Let $\mathcal{T}_{T}$ and $\mathcal{S}_{S}$ be a pseudo-t-representable t-norm and t-conorm of the first kind on $\mathcal{L}^{I}$ respectively. If at least one of $T$ and $S^{*}$ is Archimedean and both have zero-divisors, then there does not exist a scalar cardinality pattern $f_{I}$ such that $\left(f_{I}, \mathcal{T}_{T}, \mathcal{S}_{S}\right)$ satisfies the valuation property.

Proof. Assume that there exists a scalar cardinality pattern $f_{I}$ such that $\left(f_{I}, \mathcal{T}_{T}, \mathcal{S}_{S}\right)$ satisfies the valuation property. Then from Lemma 4.9 it follows that $f_{I}([0, a])$ is given by $(18)$, for all $a \in[0,1]$. 
Since $T$ has zero-divisors, there exist $a, b \in] 0,1[$ such that $T(a, b)=0$. From Theorem 4.3 if follows that

$$
0_{\mathcal{L}^{I}}=f_{I}([0,0])=f_{I}([0, T(a, b)])=f_{I}([0, \min (a, b)])=\alpha .
$$

On the other hand, since $S^{*}$ has zero-divisors, there exist $\left.a^{\prime}, b^{\prime} \in\right] 0,1[$ such that $S\left(a^{\prime}, b^{\prime}\right)=1$. Thus, using Theorem 4.3 , we obtain

$$
\left[\frac{1}{2}, \frac{1}{2}\right]=f_{I}([0,1])=f_{I}\left(\left[0, S\left(a^{\prime}, b^{\prime}\right)\right]\right)=f_{I}\left(\left[0, \max \left(a^{\prime}, b^{\prime}\right)\right]\right)=\alpha .
$$

From (19) and (20) it follows that

$$
0_{\mathcal{L}^{I}}=\alpha=\left[\frac{1}{2}, \frac{1}{2}\right]
$$

which is a contradiction.

\section{The valuation property and the class $\mathcal{T}_{T}^{\prime}$}

Lemma 5.1 Let $\mathcal{T}_{T}^{\prime}$ and $\mathcal{S}_{S}^{\prime}$ be a pseudo-t-representable t-norm and t-conorm of the second kind on $\mathcal{L}^{I}$ respectively. If $\left(f_{I}, \mathcal{T}_{T}^{\prime}, \mathcal{S}_{S}^{\prime}\right)$ satisfies the valuation property, then for all $a \in L^{I}$,

$$
f_{I}\left(\left[0, a_{2}\right]\right)+f_{I}\left(\left[a_{1}, 1\right]\right)=f_{I}(a)+f_{I}([0,1]) .
$$

Proof. This follows immediately from applying the valuation property for $a \in L^{I}$ and $b=[0,1]$.

Lemma 5.2 Let $\mathcal{T}_{T}^{\prime}$ and $\mathcal{S}_{S}^{\prime}$ be a pseudo-t-representable t-norm and t-conorm of the second kind on $\mathcal{L}^{I}$ respectively. If $\left(f_{I}, \mathcal{T}_{T}^{\prime}, \mathcal{S}_{S}^{\prime}\right)$ satisfies the valuation property, then, for all $a_{1}, a_{2}, b_{1}, b_{2}$ in $[0,1]$,

$$
\begin{aligned}
& f_{I}\left(\left[0, T\left(a_{2}, b_{2}\right)\right]\right)=f_{I}\left(\left[0, \min \left(a_{2}, b_{2}\right)\right]\right), \\
& f_{I}\left(\left[S\left(a_{1}, b_{1}\right), 1\right]\right)=f_{I}\left(\left[\max \left(a_{1}, b_{1}\right), 1\right]\right) .
\end{aligned}
$$

Proof. Let $a=\left[0, a_{2}\right]$ and $b=\left[0, b_{2}\right]$ in $L^{I}$. Then from the valuation property it follows that

$$
f_{I}\left(\left[0, T\left(a_{2}, b_{2}\right)\right]\right)+f_{I}\left(\left[0, \max \left(a_{2}, b_{2}\right)\right]\right)=f_{I}\left(\left[0, a_{2}\right]\right)+f_{I}\left(\left[0, b_{2}\right]\right) .
$$

If $a_{2} \leq b_{2}$, then it follows that

$$
f_{I}\left(\left[0, T\left(a_{2}, b_{2}\right)\right]\right)+f_{I}\left(\left[0, b_{2}\right]\right)=f_{I}\left(\left[0, a_{2}\right]\right)+f_{I}\left(\left[0, b_{2}\right]\right),
$$


or, equivalently (since from $x+z=y+z$ it follows that $x=y$, for all $x, y, z$ in $\left.L^{I}\right)$

$$
f_{I}\left(\left[0, T\left(a_{2}, b_{2}\right)\right]\right)=f_{I}\left(\left[0, a_{2}\right]\right)=f_{I}\left(\left[0, \min \left(a_{2}, b_{2}\right)\right]\right) .
$$

Let $a=\left[a_{1}, 1\right]$ and $b=\left[b_{1}, 1\right]$ in $L^{I}$. Then from the valuation property it follows that

$$
f_{I}\left(\left[\min \left(a_{1}, b_{1}\right), 1\right]\right)+f_{I}\left(\left[S\left(a_{1}, b_{1}\right), 1\right]\right)=f_{I}\left(\left[a_{1}, 1\right]\right)+f_{I}\left(\left[b_{1}, 1\right]\right) .
$$

If $a_{1} \leq b_{1}$, then it follows that

$$
f_{I}\left(\left[a_{1}, 1\right]\right)+f_{I}\left(\left[S\left(a_{1}, b_{1}\right), 1\right]\right)=f_{I}\left(\left[a_{1}, 1\right]\right)+f_{I}\left(\left[b_{1}, 1\right]\right),
$$

or, equivalently,

$$
f_{I}\left(\left[S\left(a_{1}, b_{1}\right), 1\right]\right)=f_{I}\left(\left[b_{1}, 1\right]\right)=f_{I}\left(\left[\max \left(a_{1}, b_{1}\right), 1\right]\right) .
$$

Lemma 5.3 Let $\mathcal{T}_{T}^{\prime}$ and $\mathcal{S}_{S}^{\prime}$ be a pseudo-t-representable t-norm and t-conorm of the second kind on $\mathcal{L}^{I}$ respectively. If $\left(f_{I}, \mathcal{T}_{T}^{\prime}, \mathcal{S}_{S}^{\prime}\right)$ satisfies the valuation property, then, for all $a_{1}, a_{2}, b_{1}, b_{2}$ in $[0,1]$,

$$
\begin{aligned}
& f_{I}\left(\left[T\left(a_{1}, b_{1}\right), 1\right]\right)=f_{I}\left(\left[\min \left(a_{1}, b_{1}\right), 1\right]\right), \\
& f_{I}\left(\left[0, S\left(a_{2}, b_{2}\right)\right]\right)=f_{I}\left(\left[0, \max \left(a_{2}, b_{2}\right)\right]\right) .
\end{aligned}
$$

Proof. Let $a_{2}, b_{2}$ in $[0,1]$ be arbitrary. Assume that $a_{2} \leq b_{2}$. Then from the valuation property it follows that

$$
f_{I}\left(\left[0, T\left(b_{2}, b_{2}\right)\right]\right)+f_{I}\left(\left[a_{2}, S\left(a_{2}, b_{2}\right)\right]\right)=f_{I}\left(\left[0, b_{2}\right]\right)+f_{I}\left(\left[a_{2}, b_{2}\right]\right) .
$$

Adding $f_{I}([0,1])$ to both sides of this equality, we obtain, using Lemmas 5.1 and 5.2 , that

$f_{I}\left(\left[0, \min \left(b_{2}, b_{2}\right)\right]\right)+f_{I}\left(\left[0, S\left(a_{2}, b_{2}\right)\right]\right)+f_{I}\left(\left[a_{2}, 1\right]\right)=f_{I}\left(\left[0, b_{2}\right]\right)+f_{I}\left(\left[0, b_{2}\right]\right)+f_{I}\left(\left[a_{2}, 1\right]\right)$, or, equivalently,

$$
f_{I}\left(\left[0, S\left(a_{2}, b_{2}\right)\right]\right)=f_{I}\left(\left[0, b_{2}\right]\right)=f_{I}\left(\left[0, \max \left(a_{2}, b_{2}\right)\right]\right) .
$$

Let now arbitrarily $a_{1}, b_{1}$ in $[0,1]$. Assume that $a_{1} \leq b_{1}$. Then from the valuation property it follows that

$$
f_{I}\left(\left[T\left(a_{1}, b_{1}\right), b_{1}\right]\right)+f_{I}\left(\left[S\left(a_{1}, a_{1}\right), 1\right]\right)=f_{I}\left(\left[a_{1}, b_{1}\right]\right)+f_{I}\left(\left[a_{1}, 1\right]\right) .
$$

Adding $f_{I}([0,1])$ to both sides of this equality, we obtain, using Lemmas 5.1 and 5.2 , that

$f_{I}\left(\left[0, b_{1}\right]\right)+f_{I}\left(\left[T\left(a_{1}, b_{1}\right), 1\right]\right)+f_{I}\left(\left[\max \left(a_{1}, a_{1}\right), 1\right]\right)=f_{I}\left(\left[0, b_{1}\right]\right)+f_{I}\left(\left[a_{1}, 1\right]\right)+f_{I}\left(\left[a_{1}, 1\right]\right)$, or, equivalently,

$$
f_{I}\left(\left[T\left(a_{1}, b_{1}\right), 1\right]\right)=f_{I}\left(\left[a_{1}, 1\right]\right)=f_{I}\left(\left[\min \left(a_{1}, b_{1}\right), 1\right]\right) .
$$


Theorem 5.4 Let $\mathcal{T}_{T}^{\prime}$ and $\mathcal{S}_{S}^{\prime}$ be a pseudo-t-representable $t$-norm and $t$-conorm of the second kind on $\mathcal{L}^{I}$ respectively. Then $\left(f_{I}, \mathcal{T}_{T}^{\prime}, \mathcal{S}_{S}^{\prime}\right)$ satisfies the valuation property iff

(i) for all $a \in L^{I}$,

$$
f_{I}\left(\left[0, a_{2}\right]\right)+f_{I}\left(\left[a_{1}, 1\right]\right)=f_{I}(a)+f_{I}([0,1]) .
$$

(ii) for all $a_{1}, a_{2}, b_{1}, b_{2}$ in $[0,1]$,

$$
\begin{aligned}
& f_{I}\left(\left[T\left(a_{1}, b_{1}\right), 1\right]\right)=f_{I}\left(\left[\min \left(a_{1}, b_{1}\right), 1\right]\right), \\
& f_{I}\left(\left[S\left(a_{1}, b_{1}\right), 1\right]\right)=f_{I}\left(\left[\max \left(a_{1}, b_{1}\right), 1\right]\right), \\
& f_{I}\left(\left[0, T\left(a_{2}, b_{2}\right)\right]\right)=f_{I}\left(\left[0, \min \left(a_{2}, b_{2}\right)\right]\right), \\
& f_{I}\left(\left[0, S\left(a_{2}, b_{2}\right)\right]\right)=f_{I}\left(\left[0, \max \left(a_{2}, b_{2}\right)\right]\right) .
\end{aligned}
$$

Proof. From Lemmas 5.1 and 5.2 it follows that if $\left(f_{I}, \mathcal{T}_{T}^{\prime}, \mathcal{S}_{S}^{\prime}\right)$ satisfies the valuation property, then (i) and (ii) hold.

Assume conversely that (i) and (ii) hold and let us choose arbitrarily $x, y$ in $L^{I}$. Then, using (i), (2) is equivalent to

$$
\begin{aligned}
& f_{I}\left(\left[0, T\left(x_{2}, y_{2}\right)\right]\right)+f_{I}\left(\left[\min \left(T\left(x_{1}, y_{2}\right), T\left(x_{2}, y_{1}\right)\right), 1\right]\right) \\
& +f_{I}\left(\left[0, \max \left(S\left(x_{1}, y_{2}\right), S\left(x_{2}, y_{1}\right)\right)\right]\right)+f_{I}\left(\left[S\left(x_{1}, y_{1}\right), 1\right]\right) \\
& =f_{I}\left(\left[0, x_{2}\right]\right)+f_{I}\left(\left[x_{1}, 1\right]\right)+f_{I}\left(\left[0, y_{2}\right]\right)+f_{I}\left(\left[y_{1}, 1\right]\right),
\end{aligned}
$$

or, using (ii), equivalent to

$$
\begin{aligned}
& f_{I}\left(\left[0, \min \left(x_{2}, y_{2}\right)\right]\right)+f_{I}\left(\left[\min \left(T\left(x_{1}, y_{2}\right), T\left(x_{2}, y_{1}\right)\right), 1\right]\right) \\
& +f_{I}\left(\left[0, \max \left(S\left(x_{1}, y_{2}\right), S\left(x_{2}, y_{1}\right)\right)\right]\right)+f_{I}\left(\left[\max \left(x_{1}, y_{1}\right), 1\right]\right) \\
& =f_{I}\left(\left[0, x_{2}\right]\right)+f_{I}\left(\left[x_{1}, 1\right]\right)+f_{I}\left(\left[0, y_{2}\right]\right)+f_{I}\left(\left[y_{1}, 1\right]\right) .
\end{aligned}
$$

We consider the following cases.

- If $x_{2} \leq y_{2}$ and $x_{1} \geq y_{1}$, then (2) is equivalent to

$$
\begin{aligned}
& f_{I}\left(\left[0, x_{2}\right]\right)+f_{I}\left(\left[T\left(x_{2}, y_{1}\right), 1\right]\right)+f_{I}\left(\left[0, S\left(x_{1}, y_{2}\right)\right]\right)+f_{I}\left(\left[x_{1}, 1\right]\right) \\
& =f_{I}\left(\left[0, x_{2}\right]\right)+f_{I}\left(\left[x_{1}, 1\right]\right)+f_{I}\left(\left[0, y_{2}\right]\right)+f_{I}\left(\left[y_{1}, 1\right]\right),
\end{aligned}
$$

or, equivalently,

$$
f_{I}\left(\left[\min \left(x_{2}, y_{1}\right), 1\right]\right)+f_{I}\left(\left[0, \max \left(x_{1}, y_{2}\right)\right]\right)=f_{I}\left(\left[0, y_{2}\right]\right)+f_{I}\left(\left[y_{1}, 1\right]\right),
$$

which holds, because $y_{1} \leq x_{1} \leq x_{2}$ and $x_{1} \leq x_{2} \leq y_{2}$.

- If $x_{2} \leq y_{2}$ and $x_{1} \leq y_{1}$, then (2) is equivalent to

$$
\begin{aligned}
& f_{I}\left(\left[0, x_{2}\right]\right)+f_{I}\left(\left[\min \left(T\left(x_{1}, y_{2}\right), T\left(x_{2}, y_{1}\right)\right), 1\right]\right) \\
& +f_{I}\left(\left[0, \max \left(S\left(x_{1}, y_{2}\right), S\left(x_{2}, y_{1}\right)\right)\right]\right)+f_{I}\left(\left[y_{1}, 1\right]\right) \\
& =f_{I}\left(\left[0, x_{2}\right]\right)+f_{I}\left(\left[x_{1}, 1\right]\right)+f_{I}\left(\left[0, y_{2}\right]\right)+f_{I}\left(\left[y_{1}, 1\right]\right),
\end{aligned}
$$


or, equivalently,

$$
\begin{aligned}
& f_{I}\left(\left[\min \left(T\left(x_{1}, y_{2}\right), T\left(x_{2}, y_{1}\right)\right), 1\right]\right)+f_{I}\left(\left[0, \max \left(S\left(x_{1}, y_{2}\right), S\left(x_{2}, y_{1}\right)\right)\right]\right) \\
& =f_{I}\left(\left[x_{1}, 1\right]\right)+f_{I}\left(\left[0, y_{2}\right]\right) .
\end{aligned}
$$

Using (ii) and using the fact that $x_{1} \leq y_{1}$ and $x_{2} \leq y_{2}$, we obtain that

$$
\begin{aligned}
f_{I}\left(\left[\min \left(T\left(x_{1}, y_{2}\right), T\left(x_{2}, y_{1}\right)\right), 1\right]\right) & =f_{I}\left(\left[T\left(T\left(x_{1}, y_{2}\right), T\left(x_{2}, y_{1}\right)\right), 1\right]\right) \\
& =f_{I}\left(\left[T\left(T\left(x_{1}, x_{2}\right), T\left(y_{1}, y_{2}\right)\right), 1\right]\right) \\
& =f_{I}\left(\left[\min \left(T\left(x_{1}, x_{2}\right), T\left(y_{1}, y_{2}\right)\right), 1\right]\right) \\
& =f_{I}\left(\left[T\left(x_{1}, x_{2}\right), 1\right]\right) \\
& =f_{I}\left(\left[\min \left(x_{1}, x_{2}\right), 1\right]\right) \\
& =f_{I}\left(\left[x_{1}, 1\right]\right) .
\end{aligned}
$$

Similarly, we find

$$
\begin{aligned}
f_{I}\left(\left[0, \max \left(S\left(x_{1}, y_{2}\right), S\left(x_{2}, y_{1}\right)\right)\right]\right) & =f_{I}\left(\left[0, S\left(S\left(x_{1}, y_{2}\right), S\left(x_{2}, y_{1}\right)\right)\right]\right) \\
& =f_{I}\left(\left[0, S\left(S\left(x_{1}, x_{2}\right), S\left(y_{1}, y_{2}\right)\right)\right]\right) \\
& =f_{I}\left(\left[0, \max \left(S\left(x_{1}, x_{2}\right), S\left(y_{1}, y_{2}\right)\right)\right]\right) \\
& =f_{I}\left(\left[0, S\left(y_{1}, y_{2}\right)\right]\right) \\
& =f_{I}\left(\left[0, \max \left(y_{1}, y_{2}\right)\right]\right) \\
& =f_{I}\left(\left[0, y_{2}\right]\right) .
\end{aligned}
$$

Hence (21) holds.

The other cases are shown in a similar way.

Note that from Theorem 5.4 it follows that in the case that $\mathcal{T}$ and $\mathcal{S}$ are pseudo-t-representable of the second kind, if $\left(f_{I}, \mathcal{T}, \mathcal{S}\right)$ satisfies the valuation property, then $f_{I}$ is completely determined by the values of $f_{I}([0, a])$ and $f_{I}([a, 1])$, for all $a \in[0,1]$. In the case that the representant of one of $\mathcal{T}$ and $\mathcal{S}$ is Archimedean, we can find even stronger results. First we prove some lemmas.

Lemma 5.5 Let $\mathcal{T}_{T}^{\prime}$ and $\mathcal{S}_{S}^{\prime}$ be a pseudo-t-representable t-norm and t-conorm of the second kind on $\mathcal{L}^{I}$ respectively. If at least one of $T$ and $S^{*}$ is Archimedean and $\left(f_{I}, \mathcal{T}_{T}^{\prime}, \mathcal{S}_{S}^{\prime}\right)$ satisfies the valuation property, then for all $a_{1}, a_{2}, b_{1}, b_{2}$ in $] 0,1[$ such that $a_{1} \leq a_{2}$ and $b_{1} \leq b_{2}$,

$$
\begin{aligned}
f_{I}\left(\left[0, a_{2}\right]\right) & =f_{I}\left(\left[0, b_{2}\right]\right), \\
f_{I}\left(\left[a_{1}, 1\right]\right) & =f_{I}\left(\left[b_{1}, 1\right]\right), \\
f_{I}\left(\left[a_{1}, a_{2}\right]\right) & =f_{I}\left(\left[b_{1}, b_{2}\right]\right) .
\end{aligned}
$$

Proof. Assume that $T$ or $S^{*}$ is Archimedean and $\left(f_{I}, \mathcal{T}_{T}^{\prime}, \mathcal{S}_{S}^{\prime}\right)$ satisfies the valuation property. Similarly as in the proof of Lemma 4.8, we find using Theorem $5.4\left(\right.$ ii) that $f_{I}([0, a])=f_{I}([0, b])$, for all $a, b$ in $] 0,1[$. 
In a similar way we find that $f_{I}([a, 1])=f_{I}([b, 1])$ for all $a, b$ in $] 0,1[$. Let now arbitrarily $a, b$ in $L^{I}$ such that $0<\min \left(a_{1}, b_{1}\right)$ and $\max \left(a_{2}, b_{2}\right)<1$. From Theorem 5.4 it follows that

$$
\begin{aligned}
f_{I}(a)+f_{I}([0,1]) & =f_{I}\left(\left[0, a_{2}\right]\right)+f_{I}\left(\left[a_{1}, 1\right]\right) \\
& =f_{I}\left(\left[0, b_{2}\right]\right)+f_{I}\left(\left[b_{1}, 1\right]\right) \\
& =f_{I}(b)+f_{I}([0,1]),
\end{aligned}
$$

which implies that $f_{I}(a)=f_{I}(b)$.

Lemma 5.6 Let $\mathcal{T}_{T}^{\prime}$ and $\mathcal{S}_{S}^{\prime}$ be a pseudo-t-representable t-norm and t-conorm of the second kind on $\mathcal{L}^{I}$ respectively. If at least one of $T$ and $S^{*}$ is Archimedean and $\left(f_{I}, \mathcal{T}_{T}^{\prime}, \mathcal{S}_{S}^{\prime}\right)$ satisfies the valuation property, then there exist $\alpha, \beta, \gamma$ in $L^{I}$ satisfying $\alpha \leq_{L^{I}} \beta \leq_{L^{I}} \gamma$ such that, for all $a \in L^{I}$,

$$
f_{I}(a)= \begin{cases}0_{\mathcal{L}^{I}}, & \text { if } a=0_{\mathcal{L}^{I}}, \\ \beta, & \text { if } a=[0,1] \\ 1_{\mathcal{L}^{I}}, & \text { if } a=1_{\mathcal{L}^{I}}, \\ \alpha, & \text { if } \left.a_{1}=0 \text { and } a_{2} \in\right] 0,1[, \\ \gamma, & \text { if } \left.a_{1} \in\right] 0,1\left[\text { and } a_{2}=1,\right. \\ {\left[\alpha_{1}+\gamma_{1}-\beta_{1}, \alpha_{2}+\gamma_{2}-\beta_{2}\right],} & \text { else. }\end{cases}
$$

Proof. From the definition of scalar cardinality pattern it follows that $f_{I}\left(0_{\mathcal{L}^{I}}\right)=$ $0_{\mathcal{L}^{I}}$ and $f_{I}\left(1_{\mathcal{L}^{I}}\right)=1_{\mathcal{L}^{I}}$.

From Lemma 5.5 it follows that there exist $\alpha \in L^{I}$ and $\gamma \in L^{I}$ such that $f_{I}([0, a])=\alpha$ and $f_{I}([a, 1])=\gamma$, for all $\left.a \in\right] 0,1\left[\right.$. Let $\beta=f_{I}([0,1])$. Since $f_{I}$ is increasing, $f_{I}([0, a]) \leq_{L^{I}} f_{I}([0,1]) \leq_{L^{I}} f_{I}([a, 1])$, so $\alpha \leq_{L^{I}} \beta \leq_{L^{I}} \gamma$. Using Theorem 5.4(i) it is easy to see that $f_{I}(a)=\left[\alpha_{1}+\gamma_{1}-\beta_{1}, \alpha_{2}+\gamma_{2}-\beta_{2}\right]$ for all $a \in L^{I}$ such that $0<a_{1} \leq a_{2}<1$. So $f_{I}(a)$ is given by (22), for all $a \in L^{I}$.

Theorem 5.7 Let $\mathcal{T}_{T}^{\prime}$ and $\mathcal{S}_{S}^{\prime}$ be a pseudo-t-representable $t$-norm and $t$-conorm of the second kind on $\mathcal{L}^{I}$ respectively. If at least one of $T$ and $S^{*}$ is Archimedean and at least one of them does not have zero-divisors, then $\left(f_{I}, \mathcal{T}_{T}^{\prime}, \mathcal{S}_{S}^{\prime}\right)$ satisfies the valuation property iff there exist $\alpha, \beta, \gamma$ in $L^{I}$ with $\alpha \leq_{L^{I}} \beta \leq_{L^{I}} \gamma$ such that $f_{I}(a)$ is given by (22), for all $a \in L^{I}$.

Moreover, if $T$ has zero-divisors, then $\alpha=0_{\mathcal{L}^{I}}$ and $\beta=\gamma$, and if $S^{*}$ has zero-divisors, then $\alpha=\beta$ and $\gamma=1$.

Proof. Assume first that $\left(f_{I}, \mathcal{T}_{T}^{\prime}, \mathcal{S}_{S}^{\prime}\right)$ satisfies the valuation property. From Lemma 5.6 it follows that $f_{I}(a)$ is given by (22), for all $a \in L^{I}$.

Assume conversely that there exist $\alpha, \beta, \gamma$ in $L^{I}$ such that $\alpha \leq_{L^{I}} \beta \leq_{L^{I}} \gamma$ and such that $f_{I}(a)$ is given by (22), for all $a \in L^{I}$. It is easy to see that $f_{I}$ is a scalar cardinality pattern. 
We prove that $\left(f_{I}, \mathcal{T}_{T}^{\prime}, \mathcal{S}_{S}^{\prime}\right)$ satisfies the valuation property. A straightforward calculation shows that $f_{I}\left(\left[0, a_{2}\right]\right)+f_{I}\left(\left[a_{1}, 1\right]\right)=f_{I}(a)+f_{I}([0,1])$, for all $a \in$ $L^{I}$. Let now $a, b$ in $[0,1]$ such that $a \leq b$ (the case $a \geq b$ is analogous). If $a=0$, then $f_{I}([0, T(a, b)])=f_{I}([0,0])=f_{I}([0, \min (a, b)])$. If $b=1$, then $f_{I}([0, T(a, b)])=f_{I}([0, a])=f_{I}([0, \min (a, b)])$. Similarly, if $a=0$ or $b=1$, then $\left.f_{I}(T(a, b), 1]\right)=f_{I}([\min (a, b), 1]), f_{I}([0, S(a, b)])=f_{I}([0, \max (a, b)])$ and $f_{I}([S(a, b), 1])=f_{I}([\max (a, b), 1])$. Assume now that $0<a \leq b<1$. Then we have the following cases.

- Neither $T$ or $S^{*}$ has zero-divisors: then $0<T(a, b)<1$ and, similarly as in the proof of Theorem 4.10, $0<S(a, b)<1$.

Hence $f_{I}([0, T(a, b)])=\alpha=f_{I}([0, \min (a, b)]), f_{I}([T(a, b), 1])=\gamma=$ $f_{I}([\min (a, b), 1]), f_{I}([0, S(a, b)])=\alpha=f_{I}([0, \max (a, b)])$ and $f_{I}([S(a, b), 1])=$ $\gamma=f_{I}([\max (a, b), 1])$.

- $T$ has zero-divisors: then from the assumptions it follows that $S^{*}$ does not have zero-divisors. So, similarly as in the previous case we find that $f_{I}([0, S(a, b)])=\alpha=f_{I}([0, \max (a, b)])$ and $f_{I}([S(a, b), 1])=\gamma=$ $f_{I}([\max (a, b), 1])$. Let $a^{\prime}, b^{\prime}$ in $] 0,1\left[\right.$ be such that $T\left(a^{\prime}, b^{\prime}\right)=0$. Then $f_{I}\left(\left[0, T\left(a^{\prime}, b^{\prime}\right)\right]\right)=f_{I}([0,0])=0_{\mathcal{L}^{I}}$, but on the other hand $f_{I}\left(\left[0, \min \left(a^{\prime}, b^{\prime}\right)\right]\right)=$ $\alpha$. Thus, if $f_{I}\left(\left[0, T\left(a^{\prime}, b^{\prime}\right)\right]\right)=f_{I}\left(\left[0, \min \left(a^{\prime}, b^{\prime}\right)\right]\right)$, then $\alpha=0_{\mathcal{L}^{I}}$. Conversely, if $\alpha=0_{\mathcal{L}^{I}}$, then for all $a, b$ in $] 0,1\left[, T(a, b)<1\right.$, so $f_{I}([0, T(a, b)])=$ $0_{\mathcal{L}^{I}}=f_{I}([0, \min (a, b)])$. Similarly $f_{I}([T(a, b), 1])=f_{I}([\min (a, b), 1])$ for all $a, b$ in $] 0,1[$ if and only if $\beta=\gamma$.

- $S^{*}$ has zero-divisors: similarly as in the previous case we find that $T$ does not have zero-divisors, $f_{I}([0, T(a, b)])=\alpha=f_{I}([0, \min (a, b)]), f_{I}([T(a, b), 1])=$ $\gamma=f_{I}([\min (a, b), 1]), f_{I}([0, S(a, b)])=f_{I}([0, \max (a, b)])$ and $f_{I}([S(a, b), 1])=$ $f_{I}([\max (a, b), 1])$ iff $\alpha=\beta$ and $\gamma=1_{\mathcal{L}^{I}}$.

From Theorem 5.4 it follows that, under the mentioned restrictions for $\alpha, \beta$ and $\gamma,\left(f_{I}, \mathcal{T}_{T}^{\prime}, \mathcal{S}_{S}^{\prime}\right)$ satisfies the valuation property.

Theorem 5.8 Let $\mathcal{T}_{T}^{\prime}$ and $\mathcal{S}_{S}^{\prime}$ be a pseudo-t-representable $t$-norm and $t$-conorm of the second kind on $\mathcal{L}^{I}$ respectively. If at least one of $T$ and $S^{*}$ is Archimedean and both have zero-divisors, then there does not exist a scalar cardinality pattern $f_{I}$ such that $\left(f_{I}, \mathcal{T}_{T}^{\prime}, \mathcal{S}_{S}^{\prime}\right)$ satisfies the valuation property.

Proof. Assume that there exists a scalar cardinality pattern $f_{I}$ such that $\left(f_{I}, \mathcal{T}_{T}^{\prime}, \mathcal{S}_{S}^{\prime}\right)$ satisfies the valuation property. Then from Lemma 5.6 it follows that $f_{I}([0, a])$ is given by $(22)$, for all $a \in[0,1]$.

Since $T$ has zero-divisors, there exist $a, b \in] 0,1[$ such that $T(a, b)=0$. From Theorem 5.4 if follows that

$$
\begin{aligned}
0_{\mathcal{L}^{I}} & =f_{I}([0,0])=f_{I}([0, T(a, b)])=f_{I}([0, \min (a, b)])=\alpha, \\
\beta & =f_{I}([0,1])=f_{I}([T(a, b), 1])=f_{I}([\min (a, b), 1])=\gamma .
\end{aligned}
$$


On the other hand, since $S^{*}$ has zero-divisors, there exist $\left.a^{\prime}, b^{\prime} \in\right] 0,1[$ such that $S\left(a^{\prime}, b^{\prime}\right)=1$. Thus, using Theorem 5.4 , we obtain

$$
\begin{aligned}
\beta & =f_{I}([0,1])=f_{I}\left(\left[0, S\left(a^{\prime}, b^{\prime}\right)\right]\right)=f_{I}\left(\left[0, \max \left(a^{\prime}, b^{\prime}\right)\right]\right)=\alpha, \\
1_{\mathcal{L}^{I}} & =f_{I}([1,1])=f_{I}\left(\left[S\left(a^{\prime}, b^{\prime}\right), 1\right]\right)=f_{I}\left(\left[\max \left(a^{\prime}, b^{\prime}\right), 1\right]\right)=\gamma .
\end{aligned}
$$

From (23) and (24) it follows that

$$
0_{\mathcal{L}^{I}}=\alpha=\beta=\gamma=1_{\mathcal{L}^{I}},
$$

which is a contradiction.

\section{The valuation property and the class $\mathcal{T}_{T_{1}, T_{2}}$}

Now we consider the case that $\mathcal{T}$ and $\mathcal{S}$ are t-representable.

Theorem 6.1 Let $\mathcal{T}_{T_{1}, T_{2}}$ and $\mathcal{S}_{S_{1}, S_{2}}$ be a t-representable t-norm and t-conorm on $\mathcal{L}^{I}$ respectively. Then $\left(f_{I}, \mathcal{T}_{T_{1}, T_{2}}, \mathcal{S}_{S_{1}, S_{2}}\right)$ satisfies the valuation property iff

(i) for all $a \in L^{I}$,

$$
f_{I}\left(\left[0, a_{2}\right]\right)+f_{I}\left(\left[a_{1}, 1\right]\right)=f_{I}(a)+f_{I}([0,1]),
$$

(ii) for all $a, b \in[0,1]$,

$$
\begin{aligned}
& f_{I}\left(\left[0, T_{2}(a, b)\right]\right)+f_{I}\left(\left[0, S_{2}(a, b)\right]\right)=f_{I}([0, a])+f_{I}([0, b]), \\
& f_{I}\left(\left[T_{1}(a, b), 1\right]\right)+f_{I}\left(\left[S_{1}(a, b), 1\right]\right)=f_{I}([a, 1])+f_{I}([b, 1]) .
\end{aligned}
$$

Proof. Assume first that $\left(f_{I}, \mathcal{T}_{T_{1}, T_{2}}, \mathcal{S}_{S_{1}, S_{2}}\right)$ satisfies the valuation property. Let $a \in L^{I}$ and $b=[0,1] \in L^{I}$, then $\mathcal{T}_{T_{1}, T_{2}}(a, b)=\left[0, a_{2}\right]$ and $\mathcal{S}_{S_{1}, S_{2}}(a, b)=\left[a_{1}, 1\right]$, so from (2) it follows that (i) holds. Let now arbitrarily $a_{2}, b_{2} \in[0,1]$, then $a=\left[0, a_{2}\right], b=\left[0, b_{2}\right] \in L^{I}$, so from (2) it follows that

$$
f_{I}\left(\left[0, T_{2}\left(a_{2}, b_{2}\right)\right]\right)+f_{I}\left(\left[0, S_{2}\left(a_{2}, b_{2}\right)\right]\right)=f_{I}\left(\left[0, a_{2}\right]\right)+f_{I}\left(\left[0, b_{2}\right]\right) .
$$

Similarly, the second equality of (ii) can be proven.

Assume conversely that (i) and (ii) hold. Then for all $a, b \in L^{I}$,

$$
\begin{aligned}
& f_{I}\left(\mathcal{T}_{T_{1}, T_{2}}(a, b)\right)+f_{I}\left(\mathcal{S}_{S_{1}, S_{2}}(a, b)\right)+f_{I}([0,1])+f_{I}([0,1]) \\
& =f_{I}\left(\left[0, T_{2}\left(a_{2}, b_{2}\right)\right]\right)+f_{I}\left(\left[0, S_{2}\left(a_{2}, b_{2}\right)\right]\right)+f_{I}\left(\left[T_{1}\left(a_{1}, b_{1}\right), 1\right]\right)+f_{I}\left(\left[S_{1}\left(a_{1}, b_{1}\right), 1\right]\right) \\
& =f_{I}\left(\left[0, a_{2}\right]\right)+f_{I}\left(\left[0, b_{2}\right]\right)+f_{I}\left(\left[a_{1}, 1\right]\right)+f_{I}\left(\left[b_{1}, 1\right]\right) \\
& =f_{I}(a)+f_{I}(b)+f_{I}([0,1])+f_{I}([0,1]) .
\end{aligned}
$$

Since from $x+z=y+z$ it follows that $x=y$, for all $x, y, z \in L^{I}$, we obtain that (2) holds.

From Theorem 6.1 it follows that in the case that $\mathcal{T}$ and $\mathcal{S}$ are t-representable, if $\left(f_{I}, \mathcal{T}, \mathcal{S}\right)$ satisfies the valuation property, then $f_{I}$ is completely determined by the value of $f_{I}([0, a])$ and $f_{I}([a, 1])$, for all $a \in[0,1]$. 
Corollary 6.2 Let $\mathcal{T}_{T_{1}, T_{2}}$ and $\mathcal{S}_{S_{1}, S_{2}}$ be a t-representable $t$-norm and $t$-conorm on $\mathcal{L}^{I}$ respectively. Then $\left(f_{I}, \mathcal{T}_{T_{1}, T_{2}}, \mathcal{S}_{S_{1}, S_{2}}\right)$ satisfies the valuation property iff there exist an element $\alpha \in L^{I}$ and scalar cardinality patterns $f_{11}, f_{12}, f_{21}$ and $f_{22}$ on the unit interval such that

(i) for all $a \in L^{I}$,

$$
\begin{aligned}
f_{I}(a)= & {\left[\alpha_{1} f_{21}\left(a_{2}\right)+\left(1-\alpha_{1}\right) f_{11}\left(a_{1}\right),\right.} \\
& \left.\alpha_{2} f_{22}\left(a_{2}\right)+\left(1-\alpha_{2}\right) f_{12}\left(a_{1}\right)\right],
\end{aligned}
$$

(ii) $\left(f_{11}, T_{1}, S_{1}\right),\left(f_{12}, T_{1}, S_{1}\right),\left(f_{21}, T_{2}, S_{2}\right)$ and $\left(f_{22}, T_{2}, S_{2}\right)$ satisfy the valuation property.

Proof. Assume first that $\left(f_{I}, \mathcal{T}_{T_{1}, T_{2}}, \mathcal{S}_{S_{1}, S_{2}}\right)$ satisfies the valuation property. Let $\alpha=f_{I}([0,1])$. Define for all $a \in[0,1]$,

$$
\begin{aligned}
& f_{11}(a)=\frac{\left(f_{I}([a, 1])\right)_{1}-\left(f_{I}([0,1])\right)_{1}}{1-\left(f_{I}([0,1])\right)_{1}}, \text { if }\left(f_{I}([0,1])\right)_{1}<1, \\
& f_{12}(a)=\frac{\left(f_{I}([a, 1])\right)_{2}-\left(f_{I}([0,1])\right)_{2}}{1-\left(f_{I}([0,1])\right)_{2}}, \text { if }\left(f_{I}([0,1])\right)_{2}<1, \\
& f_{21}(a)=\frac{\left(f_{I}([0, a])\right)_{1}}{\left(f_{I}([0,1])\right)_{1}}, \text { if }\left(f_{I}([0,1])\right)_{1}>0, \\
& f_{22}(a)=\frac{\left(f_{I}([0, a])\right)_{2}}{\left(f_{I}([0,1])\right)_{2}}, \text { if }\left(f_{I}([0,1])\right)_{2}>0 .
\end{aligned}
$$

It is easy to verify that $f_{11}, f_{12}, f_{21}$ and $f_{22}$ are scalar cardinality patterns on the unit interval. From Theorem 6.1(ii) it follows for all $a, b \in[0,1]$ that

$$
\left(f_{I}\left(\left[0, T_{2}(a, b)\right]\right)\right)_{1}+\left(f_{I}\left(\left[0, S_{2}(a, b)\right]\right)\right)_{1}=\left(f_{I}([0, a])\right)_{1}+\left(f_{I}([0, b])\right)_{1},
$$

so

$$
f_{21}\left(T_{2}(a, b)\right)+f_{21}\left(S_{2}(a, b)\right)=f_{21}(a)+f_{21}(b),
$$

i.e. $\left(f_{21}, T_{2}, S_{2}\right)$ satisfies the valuation property. Similarly $\left(f_{11}, T_{1}, S_{1}\right),\left(f_{12}, T_{1}\right.$, $\left.S_{1}\right)$ and $\left(f_{22}, T_{2}, S_{2}\right)$ satisfy the valuation property.

Now we calculate the right hand side of (25) for all $a \in L^{I}$. We have the following cases.

- If $\left(f_{I}([0,1])\right)_{1}=0$, then the first projection of the right hand side of $(25)$ is equal to $0+f_{11}\left(a_{1}\right)=\left(f_{I}\left(\left[a_{1}, 1\right]\right)\right)_{1}$. On the other hand, from Theorem 6.1 (i) it follows that $0+\left(f_{I}\left(\left[a_{1}, 1\right]\right)\right)_{1}=\left(f_{I}(a)\right)_{1}+0$, so the first projection of the right hand side of $(25)$ is equal to $\left(f_{I}(a)\right)_{1}$.

- If $\left(f_{I}([0,1])\right)_{1}=1$, then the first projection of the right hand side of $(25)$ is equal to $f_{21}\left(a_{2}\right)+0=\left(f_{I}\left(\left[0, a_{2}\right]\right)\right)_{1}$. On the other hand, from Theorem 6.1 (i) it follows that $\left(f_{I}\left(\left[0, a_{2}\right]\right)\right)_{1}+1=\left(f_{I}(a)\right)_{1}+1$, so the first projection of the right hand side of $(25)$ is equal to $\left(f_{I}(a)\right)_{1}$. 
- $\left.\left(f_{I}([0,1])\right)_{1} \in\right] 0,1[$, then the first projection of the right hand side of (25) is equal to $\left(f_{I}([0,1])\right)_{1} f_{21}\left(a_{2}\right)+\left(1-\left(f_{I}([0,1])\right)_{1}\right) f_{11}\left(a_{1}\right)=\left(f_{I}\left(\left[0, a_{2}\right]\right)\right)_{1}+$ $\left(f_{I}\left(\left[a_{1}, 1\right]\right)\right)_{1}-\left(f_{I}([0,1])\right)_{1}$, which according to Theorem 6.1(i) is equal to $\left(f_{I}(a)\right)_{1}$.

A similar calculation shows that the second projection of the right hand side of $(25)$ is equal to $\left(f_{I}(a)\right)_{2}$. So (i) holds.

Assume conversely that there exist an element $\alpha \in L^{I}$ and scalar cardinality patterns $f_{11}, f_{12}, f_{21}$ and $f_{22}$ on the unit interval such that (ii) holds and define the mapping $f_{I}: L^{I} \rightarrow L^{I}$ by (i). A straightforward calculation shows that $f_{I}$ is a scalar cardinality pattern on $\mathcal{L}^{I}$. It only remains to show that $\left(f_{I}, \mathcal{T}_{T_{1}, T_{2}}, \mathcal{S}_{S_{1}, S_{2}}\right)$ satisfies the valuation property. We have the following cases.

- If $\alpha_{1}=0$, then $\left(f_{I}(a)\right)_{1}=f_{11}\left(a_{1}\right)$, for all $a \in L^{I}$. So, for all $a, b \in L^{I}$, we obtain that

$$
\begin{aligned}
& \left(f_{I}\left(\mathcal{T}_{T_{1}, T_{2}}(a, b)\right)+f_{I}\left(\mathcal{S}_{S_{1}, S_{2}}(a, b)\right)\right)_{1} \\
& =f_{11}\left(T_{1}\left(a_{1}, b_{1}\right)\right)+f_{11}\left(S_{1}\left(a_{1}, b_{1}\right)\right) \\
& =f_{11}\left(a_{1}\right)+f_{11}\left(b_{1}\right) \\
& =\left(f_{I}(a)+f_{I}(b)\right)_{1},
\end{aligned}
$$

using the fact that $\left(f_{11}, T_{1}, S_{1}\right)$ satisfies the valuation principle.

- If $\alpha_{1}=1$, then $\left(f_{I}(a)\right)_{1}=f_{21}\left(a_{2}\right)$, for all $a \in L^{I}$. We obtain similarly that $\left(f_{I}\left(\mathcal{T}_{T_{1}, T_{2}}(a, b)\right)+f_{I}\left(\mathcal{S}_{S_{1}, S_{2}}(a, b)\right)\right)_{1}=\left(f_{I}(a)+f_{I}(b)\right)_{1}$, for all $a, b \in L^{I}$.

- If $\left.\alpha_{1} \in\right] 0,1\left[\right.$, then for all $a, b \in L^{I}$,

$$
\begin{aligned}
& \left(f_{I}\left(\mathcal{T}_{T_{1}, T_{2}}(a, b)\right)+f_{I}\left(\mathcal{S}_{S_{1}, S_{2}}(a, b)\right)\right)_{1} \\
& =\alpha_{1} f_{21}\left(T_{2}\left(a_{2}, b_{2}\right)\right)+\left(1-\alpha_{1}\right) f_{11}\left(T_{1}\left(a_{1}, b_{1}\right)\right) \\
& \quad+\alpha_{1} f_{21}\left(S_{2}\left(a_{2}, b_{2}\right)\right)+\left(1-\alpha_{1}\right) f_{11}\left(S_{1}\left(a_{1}, b_{1}\right)\right) \\
& =\alpha_{1} f_{21}\left(a_{2}\right)+\left(1-\alpha_{1}\right) f_{11}\left(a_{1}\right)+\alpha_{1} f_{21}\left(b_{2}\right)+\left(1-\alpha_{1}\right) f_{11}\left(b_{1}\right) \\
& =\left(f_{I}(a)+f_{I}(b)\right)_{1},
\end{aligned}
$$

using the fact that $\left(f_{11}, T_{1}, S_{1}\right)$ and $\left(f_{21}, T_{2}, S_{2}\right)$ satisfy the valuation property.

Similarly we obtain that $\left(f_{I}\left(\mathcal{T}_{T_{1}, T_{2}}(a, b)\right)+f_{I}\left(\mathcal{S}_{S_{1}, S_{2}}(a, b)\right)\right)_{2}=\left(f_{I}(a)+f_{I}(b)\right)_{2}$, for all $a, b \in L^{I}$. This completes the proof. Note that for any choice of $\alpha \in L^{I}$, $f_{I}([0,1])=\alpha$.

\section{Conclusions}

We provided in this paper the characterization of cardinality patterns for which the corresponding scalar cardinalities of finite interval-valued fuzzy sets satisfy 
the valuation property with respect to different classes of t-norms and t-conorms on the lattice $\mathcal{L}^{I}$. The paper covers the t-representable t-(co)norms, the pseudo t-representable t-(co)norms of the first and second kind and the generalized pseudo-t-representable t-(co)norms. Our future research will focus on further description of cardinality patterns, t-(co)norms and negations satisfying the subadditivity property, the complementarity rule and the cartesian product rule.

The choice of the appropriate cardinality pattern is based on the intended application and therefore strictly context dependent. Obviously it goes beyond the scope of our paper. Nevertheless, we suppose that the presented characterization can increase the applicability of the cardinality theory presented in $[11]$.

\section{References}

[1] K. T. Atanassov. Intuitionistic fuzzy sets, 1983. VII ITKR's Session, Sofia (deposed in Central Sci--Technical Library of Bulg. Acad. of Sci., 1697/84) (in Bulgarian).

[2] K. T. Atanassov. Intuitionistic Fuzzy Sets. Physica-Verlag, Heidelberg, New York, 1999.

[3] J. Casasnovas and J. Torrens. Scalar cardinalities of finite fuzzy sets for t-norms and t-conorms. International Journal of Uncertainty, Fuzziness and Knowledge-Based Systems, 11(5):599-614, 2003.

[4] M. Casasnovas and J. Torrens. An axiomatic approach to fuzzy cardinalities of finite fuzzy sets. Fuzzy Sets and Systems, 133(2):193-209, 2003.

[5] B. De Baets, S. Janssens and H. De Meyer, Meta-theorems on inequalities for scalar fuzzy set cardinalities. Fuzzy Sets and Systems, 157:1463-1476, 2006.

[6] A. De Luca and S. Termini. A definition of non-probabilistic entropy in the setting of fuzzy sets theory. Inform. Control, 20:301-312, 1972.

[7] G. Deschrijver. A thorough study of the basic operators in intuitionistic fuzzy set theory. PhD thesis, Ghent University, Belgium, 2004. in Dutch.

[8] G. Deschrijver. Arithmetic operators in interval-valued fuzzy set theory. Information Sciences, 177(14):2906-2924, 2007.

[9] G. Deschrijver, C. Cornelis, and E. E. Kerre. On the representation of intuitionistic fuzzy t-norms and t-conorms. IEEE Transactions on Fuzzy Systems, 12(1):45-61, 2004.

[10] G. Deschrijver and E. E. Kerre. On the relationship between some extensions of fuzzy set theory. Fuzzy Sets and Systems, 133(2):227-235, 2003. 
[11] G. Deschrijver and P. Král. On the cardinalities of interval-valued fuzzy sets. Fuzzy Sets and Systems, 158(15):1728-1750, 2007.

[12] D. Dubois and H. Prade. Fuzzy cardinality and the modeling of imprecise quantification. Fuzzy Sets and Systems, 16(3):199-230, 1985.

[13] J. A. Goguen. L-fuzzy sets. Journal of Mathematical Analysis and Applications, 18(1):145-174, Apr 1967.

[14] S. Gottwald. A note on fuzzy cardinals. Kybernetika, 16:156-158, 1980.

[15] A. Kaufmann. Introduction to the theory of fuzzy subsets. Academic Press, New York, 1975.

[16] D. Ralescu. Cardinality, quantifiers, and the aggregation of fuzzy criteria. Fuzzy Sets and Systems, 69(3):355-365, 1995.

[17] D. Rocacher and P. Bosc. The set of fuzzy rational numbers and flexible querying. Fuzzy Sets and Systems, 155(3):317-339, 2005.

[18] E. Szmidt and J. Kacprzyk. Entropy for intuitionistic fuzzy sets. Fuzzy Sets and Systems, 118(3):467-477, 2001.

[19] M. Wygralak. Cardinalities of fuzzy sets evaluated by single cardinals. In Proc. IFSA Congress, pages 73-77. Praha, 1997.

[20] M. Wygralak. An axiomatic approach to scalar cardinalities of a fuzzy set. Fuzzy Sets and Systems, 110(2):175-176, 2000.

[21] M. Wygralak. Cardinalities of Fuzzy Sets. Springer-Verlag, Berlin, Heidelberg, New York, 2003. 